\title{
Macrozooplankton communities and environmental variables in the Barents Sea marginal ice zone in late winter and spring
}

\author{
Janne E. Søreide ${ }^{1,2,3, *}$, Haakon Hop ${ }^{1}$, Stig Falk-Petersen ${ }^{1}$, Bjørn Gulliksen ${ }^{2}$, \\ Edmond Hansen ${ }^{1}$
}

\author{
${ }^{1}$ Norwegian Polar Institute, 9296 Tromsø, Norway \\ ${ }^{2}$ Norwegian College of Fishery Science, University of Tromsø, 9037 Tromsø, Norway \\ ${ }^{3}$ Present address: Akvaplan-niva, 9296 Tromsø, Norway
}

\begin{abstract}
The horizontal distribution of macrozooplankton $(\geq 3 \mathrm{~mm}$ ) was studied with respect to changes in environmental conditions along 3 transects across the marginal ice zone (MIZ) in the NW Barents Sea during late winter (March 2000) and spring (May 1999). The water masses, which were identified by their salinity and temperature characteristics, largely determined the macrozooplankton composition, but season (late winter vs spring), geographical area (latitude and longitude) and bottom depth were also important. Almost $80 \%$ of the total macrozooplankton variability could be explained by changes in these environmental variables. Ice cover and the algal bloom situation had no significant impact on the horizontal macrozooplankton distribution early in the season (March to May). We sampled 3 main water masses: cold and less saline Arctic water (ArW), warm and saline Atlantic water (AtW) and mixtures of these (MIX); 5 distinct macrozooplankton communities were found, 2 in ArW (ArW bank and ArW slope), 2 in MIX (warm, $>1^{\circ} \mathrm{C}$, and cold, $<0^{\circ} \mathrm{C}, \mathrm{MIX}$ ) and 1 in AtW. These communities were characterised by differences in species abundance rather than differences in taxonomic composition. Numerically important macrozooplankton were Calanus glacialis, C. hyperboreus, Thysanoessa inermis and Aglantha digitale. These were also important in terms of wet biomass together with Beröe cucumis, Clione limacina and Sagitta elegans. Good indicator species for ArW were C. glacialis, C. limacina, Mertensia ovum and Parathemisto libellulla, and for AtW Thysanoessa spp. (T. inermis, T. longicaudata and T. raschii). Characteristic for MIX, i.e. the Polar Front region, was low macrozooplankton abundance, biomass and species richness compared to AtW and ArW. Seasonal variability (late winter vs spring) accounted for $21 \%$ of the total macrozooplankton variability. Particularly the herbivores showed large seasonal variability in abundance and biomass, most probably due to their seasonal migration patterns.
\end{abstract}

KEY WORDS: Macrozooplankton - Barents Sea - Marginal ice zone $\cdot$ Multivariate community analyses $\cdot$ Ordination

\section{INTRODUCTION}

The Barents Sea is an Arctic shelf sea, located between 70 to $80^{\circ} \mathrm{N}$ and 20 to $60^{\circ} \mathrm{E}$, with an average depth of $230 \mathrm{~m}$ (Zenckevitch 1963). It is a productive, highlatitude marine ecosystem that supports one of the world's richest fisheries (Zenckevitch 1963, Hamre 1994, Sakshaug et al. 1994). Most zooplankton studies in the
Barents Sea have been limited to the numerically important copepods, particularly of the genus Calanus, which may constitute up to $90 \%$ of the total zooplankton biomass during summer (e.g. Eilertsen et al. 1989, Hassel 1995, Pedersen et al. 1995, Falk-Petersen et al. 1999). Distribution and quantitative information on large zooplankton organisms ( $\geq 3 \mathrm{~mm})$, i.e. hydromedusas, siphonophores, ctenophores, chaetognaths, amphipods 
and euphausiids, are still limited. Although their contribution to the zooplankton in terms of abundance can be negligible, their role in trophodynamic processes might be very important due to their large predation impact (Falkenhaug 1991, Swanberg \& Båmstedt 1991, Dalpadado \& Skjoldal 1996, Dalpadado et al. 2001). Large variations in zooplankton abundance, biomass and structure have been recorded in the Barents Sea on seasonal, interannual and geographical scales (Hassel 1986, Skjoldal et al. 1987, Skjoldal \& Rey 1989, Arashkevich et al. 2002). Strong seasonality combined with large intra- and interannual variations in inflowing warm $\left(>1^{\circ} \mathrm{C}\right.$ ) and saline ( $\geq 35 \mathrm{PSU}$ ) Atlantic water (AtW) and, thus, ice cover, contributes to these zooplankton fluctuations, but to what extent is unknown.

The Barents Sea is a highly advective ecosystem, being connected to the Arctic Ocean in the north, the Kara Sea in the east and the Norwegian Sea in the southwest. The main inflow of AtW is from the southwest via the deeper trench between Norway and Bear Island (Bjørnøya), but some AtW (derived from the West Spitsbergen Current) also flows in from the north between Nordaustlandet and Franz Josef Land as a near-bottom current (Loeng 1991, Løyning 2000). The latter AtW is often referred to as cold AtW, because it is usually cooled and partly transformed on its way around the western and northern Spitsbergen coasts (Løyning 2000, Kaltin et al. 2002). AtW meets and mixes with the colder $\left(<0^{\circ} \mathrm{C}\right)$ and less saline $(34.3$ to 34.8 PSU) Arctic water (ArW) at the Polar Front (see Fig. 1). This topographically steered oceanic front divides the western Barents Sea into a warm, ice-free southern part and a cold, seasonally ice-covered, northern part (Loeng 1991). The maximum sea-ice extent during winter normally coincides with the Polar Front, and during late summer and autumn, the Barents Sea becomes almost ice-free (Vinje \& Kvambekk 1991). Melting of sea ice during spring and summer gives rise to strongly stratified surface waters that, in concert with increasing light levels and winter-accumulated nutrients, create conditions for intensive algal blooms (Skjoldal \& Rey 1989, Hegseth 1998, Falk-Petersen et al. 2000a). These blooms follow the receding ice edge as it moves northwards, and produce a high zooplankton abundance and biomass that subsequently influence the abundance of fishes as well as marine mammals and sea birds (Hassel et al. 1991, Nilssen et al. 1995, Mehlum et al. 1998). The intense seasonality of primary production at high latitudes means that food for herbivorous zooplankton is severely limited in winter. Many of them thus enter a non-feeding mode and overwinter at depth, utilising lipid reserves synthesised the previous summer (Tande \& Henderson 1988, Hagen \& Auel 2001). The seasonal resource limitation is one important cause for the generally delayed repro- ductive maturity and longer life span in high-latitude marine invertebrates compared to those from lower, more temperate latitudes (Clarke 1983, 1988). For instance, the generation time of the large herbivorous copepod Calanus hyperboreus ranges from $1 \mathrm{yr}$ in the Norwegian Sea to 3-4 yr in the Greenland Sea and the Arctic Ocean (Wiborg 1954, Østvedt 1955, Dawson 1978, Hirche 1997). Zooplankton populations of different zoogeographical origin (i.e. Arctic vs Atlantic) may therefore have markedly different life histories and size distributions (Mileikovsky 1970, Kwasnievski et al. 2003). Potentially, a total biomass of $660 \times 10^{3} \mathrm{t} \mathrm{C}$ of the Atlantic copepod Calanus finmarchicus can be transported with AtW into the Barents Sea (Pedersen 1995). In comparison, the endemic production of $C$. finmarchicus during the same period is about $100 \times$ $10^{3}$ t C (Pedersen 1995). The distribution of ArW and AtW, and their mixing (MIX), may therefore have a major influence on zooplankton distribution and abundance in the Barents Sea (Tande et al. 1985, Hassel 1995, Pedersen 1995, Falk-Petersen et al. 1999).

In the present study, the horizontal community pattern of macrozooplankton across the marginal ice zone (MIZ) in the Barents Sea, from dense pack-ice to open water, was determined during late winter (March) and spring (May). Species were divided into developmental stages (copepods) and size-classes (abundant non-copepod species) and subsequently into herbivores, omnivores and carnivores in order to study community structures. Multivariate statistics were used to relate the major community patterns to the environmental variables: ice cover, salinity, temperature, bottom depth, latitude, longitude and stage of the algal bloom.

\section{MATERIALS AND METHODS}

Transect description. The data were collected during 2 cruises with the ice-strengthened RV 'Lance' from 1 to 26 May 1999 and 8 to 23 March 2000. Stations were located in areas with different ice conditions (close pack-ice, open drift-ice and open water) within the MIZ. Transects began as far north into the pack ice as ice conditions allowed, and moved southwards into less dense ice (see Table 1, Fig. 1). In May, 2 transects were followed in the MIZ: Transect A ( 7 to 14 May) in the central Barents Sea started at the southern Great Bank $(<200 \mathrm{~m})$ and ended in the deeper Hopen Trench ( $\sim 300 \mathrm{~m})$, and Transect B (16 to 24 May) was further west, starting north of Hopen ( 200 m) and extending south into the shallower parts of the Spitsbergen Bank $(<100 \mathrm{~m})$. The last station on each transect (Stns A5 and B5) was supplemented by an oceanographical transect (including 15 stations) along the ice edge 
across the Hopen Trench (5 to 7 May) (Hop \& FalkPetersen 2003). In March, another transect was sampled in the MIZ in the central Barents Sea: Transect C (16 to 20 March), starting from the NW Great Bank $(<180 \mathrm{~m})$ and ending up in the Hopen Trench $(\sim 300 \mathrm{~m})$. A second transect across MIZ near Hopen in March was cancelled due to bad weather.

Hydrography, ice characteristics and chlorophyll a. Salinity and temperature were recorded at each station, within $1 \mathrm{~h}$ before the macrozooplankton sampling, with a Sea-Bird Electronics SBE 911+ CTD sonde. The properties of the water masses (Arctic vs Atlantic) with respect to salinity and temperature were obtained from recent literature (Loeng 1991, Harris et al. 1998). The CTD data were assigned to a regular grid in MATLAB v6.5, and the salinity-temperature field between the individual stations was calculated by linear interpolation.

Ice concentration, ice thickness and floe size were observed and noted every third hour from the ship's bridge by trained personnel, and documented by digital pictures (Hop \& Falk-Petersen 2003). Estimation of ice concentration (in tenths) was based on schematic diagrams from the National Oceanic and Atmospheric Administration (NOAA 2001). The chlorophyll a biomass (integrated over 0 to $50 \mathrm{~m}$ ) and the stage of the algal bloom in May 1999 and March 2000 were obtained from Engelsen et al. (2002) and E. N. Hegseth (unpubl.). Recordings from a Sea Tech fluorometer attached to the CTD were used to estimate the approximate algal biomass at stations for which chlorophyll $a$ samples were missing (Stns A3, B5, C2 and C3). The algal bloom situation was determined from the vertical distribution of chlorophyll a (mg chl $\left.a \mathrm{~m}^{-3}\right)$.

Zooplankton sampling. Since trawling is risky and often impossible in the MIZ, vertical hauls with a large Nansen closing net (Macrozooplankton net, Hydro-Bios) specially made for this study were used to ensure a sufficient volume of filtered water. The net had a mouth opening of $2.01 \mathrm{~m}^{2}$ and a $7.0 \mathrm{~m}$ long net bag with $1.55 \mathrm{~mm}$ mesh. The sampling depth was calculated from the wire angle and wire length, whereas the volume of filtered water was calculated using a flow meter (digital flow meter, Model 438 110, Hydro-Bios). Comparisons of flowmeter readings and the sampled volume of water calculated from the wire length and mouth opening gave filtration efficiency close to $100 \%(100.8 \pm 1.7 \%)$, and $100 \%$ filtration efficiency was assumed. We sampled 3 replicates per station, vertically, from ca. $20 \mathrm{~m}$ above the bottom to the surface, at a hauling speed of $\sim 1 \mathrm{~m} \mathrm{~s}^{-1}$. All sampling was performed during daylight.

In March 2000, 2 depth strata were sampled at Stns C1 and C2: 3 samples below and 3 above the pycnocline, which was determined from the CTD profile before sampling. The depth-stratified samples were subse- quently combined in the analyses of horizontal macrozooplankton distribution. Zooplankton was preserved in $4 \%$ buffered formaldehyde solution immediately after sampling and was analysed within 1 to 3 mo.

Zooplankton smaller than adult Calanus glacialis $(<3 \mathrm{~mm})$ were poorly sampled $(<10 \%)$ with the macrozooplankton net compared to the multinet (opening $0.25 \mathrm{~m}^{2}$, mesh size $180 \mu \mathrm{m}$ ) used in a complementary project during the May 1999 cruise (Hop \& FalkPetersen 2003). Therefore, only zooplankton $\geq 3 \mathrm{~mm}$ were defined as macrozooplankton in this study (this excluded the copepods Metridia longa, and C. finmarchicus and smaller copepodites of $C$. glacialis $[<\mathrm{CVI}], C$. hyperboreus $[<\mathrm{CVI}]$ and Pareuchaetha spp. $[<\mathrm{CVI}])$. Half of the numbers $(51.6 \pm 17.1 \%)$ of adult C. glacialis, the smallest copepod defined as macrozooplankton in this study, were caught by the macrozooplankton net, whereas adults of the large C. hyperboreus were caught in slightly higher numbers $(119.0 \pm 15.7 \%)$ than in the multinet.

Zooplankton analysis. All specimens were counted and measured to the nearest millimetre under a stereomicroscope equipped with a calibrated occular micrometer, and identified to species level when possible. Different stages of Calanus species were identified by prosome length (Unstad \& Tande 1991). Euphausiids were classified as juvenile or adult by secondary sexual characteristics, the petasma of males and the thelycum of females (Einarsson 1945). Individuals with no visible secondary sexual characteristics were classified as juveniles.

Total body length (TL) of fully extended krill and amphipods were measured along the dorsal line from the tip of the rostrum (from the front of the head in amphipods) to the end of the telson. The TL of chaetognaths was measured from the top of the head to the end of the tail (excluding the tail fin) and the TL of the pteropod Clione limacina was measured from the top of the head (excluding anterior antenna) to the end of the body. In the ctenophores Beröe cucumis and Mertensia ovum, the TL and oral-aboral length were measured, respectively. Live lengths of ctenophores and C. limacina were preferred, since their body lengths shrank considerably after preservation. In May 1999, live length of C. limacina $<10 \mathrm{~mm}$ and ctenophores was not measured, only body length after preservation. Live lengths $\left(\mathrm{TL}_{\text {live }}\right)$ of these individuals were therefore calculated from formaldehyde-preserved $\left(\mathrm{TL}_{\text {formalin }}\right.$ - - live length regressions obtained from individuals collected in March 2000: $M$. ovum: $\mathrm{TL}_{\text {live }}=2.104 \mathrm{TL}_{\text {formalin }}+3.897\left(\mathrm{n}=7, \mathrm{TL}_{\text {live }}\right.$ range $\left.=13-40 \mathrm{~mm}, \mathrm{r}^{2}=0.70, F_{1,5}=11.7, \mathrm{p}=0.018\right) ;$ B. cucumis: $\mathrm{TL}_{\text {live }}=2.509 \mathrm{TL}_{\text {formalin }}\left(\mathrm{n}=12, \mathrm{TL}_{\text {live }}\right.$ range $=10-58 \mathrm{~mm}$, $\left.\mathrm{r}^{2}=0.87, F_{1,10}=69.9, \mathrm{p}<0.001\right) ; C$. limacina: $\mathrm{TL}_{\mathrm{live}}=1.732$ $\mathrm{TL}_{\text {formalin }}-1.484 \mathrm{(n}=29, \mathrm{TL}_{\text {live }}$ range $=10-60 \mathrm{~mm}, \mathrm{r}^{2}=0.73$, $\left.F_{1,27}=74.1, \mathrm{p}<0.001\right)$. 
The total bell height of Aglantha digitale was measured from the tip of the manubrium to its rim (Fig. 2 in Matthews \& Hestad 1977). This dimension does not shrink by fixation in formaldehyde (Rasmussen 1971).

Wet weights $( \pm 0.1 \mathrm{mg})$ were obtained from formaldehyde-preserved individuals. The specimens were blotted quickly on filter paper to remove water adhering to the body surface prior to weighing. Taxa with small-sized individuals (copepods, Sarsia spp. and Aeginopsis laurentii) were pooled before weighing. Depth-integrated (bottom-to-surface) species abundance and biomass were analysed and calculated independently for each replicate.

Data analyses. In terms of abundance (individuals $\mathrm{m}^{-2}$ ), the commonly occurring macrozooplankton species were divided into small, medium and large individuals, but in terms of wet biomass $\left(\mathrm{g} \mathrm{m}^{-2}\right)$ they were not. Replicate variability in species abundance and biomass was examined by the similarity analysis program SIMPER and non-metrical multi-dimensional scaling (MDS) in PRIMER v5.0 (Clarke \& Warwick 1994, Clarke \& Gorley 2001). The analyses were based on Bray-Curtis similarities between sample pairs (Bray $\&$ Curtis 1957) on $\log (x+1)$-transformed abundance and biomass data.

To study the effect of several environmental variables simultaneously on a multitude of species, indirect (correspondence analysis, CA) and direct (canonical correspondence analysis, CCA) gradient analyses were performed using CANOCO for Windows v4.0 (ter Braak \& Smilauer 1998). CA and CCA are based on the assumption of a non-linear species-environment response, and are applied to relative, not absolute, species data (ter Braak \& Smilauer 1998). Our species data contained $50 \%$ zeros and showed a moderate unimodal response (gradient length $\sim 2$ SD). Principal correspondence analysis, which assumes a linear species-environment response, was tested, but the nonlinear methods fitted our data slightly better. Species data with many zeros are often best analysed with unimodal methods (ter Braak \& Verdonschot 1995, ter Braak \& Smilauer 1998). The mean species data per station were used in the CA and CCA. The species data were $\log (x+1)$-transformed, and rarely occurring taxa were made redundant (i.e. passive) to prevent them from greatly influencing the analyses (ter Braak \& Smilauer 1998). CA was used to determine the major macrozooplankton distribution patterns, whereas CCA was used to rank the environmental variables according to their relative importance by applying a stepwise procedure called 'forward selection' (ter Braak \& Verdenschot 1995). Only those environmental variables that significantly explained the species patterns in the CCA (Monte Carlo test run with 999 unrestricted permutations, $\mathrm{p}<0.05)$ were used in the CA analyses. The statistical significance of the relationship between species and the whole set of environmental variables, given the covariables (when present), was tested by a Monte Carlo permutation test using 'the global permutation test' in the CANOCOpackage (ter Braak \& Smilauer 1998). Seasonal macrozooplankton variability was removed by identifying season as covariable (partial correspondence analysis) (ter Braak \& Verdonschot 1995). The environmental variables salinity and temperature were determined from the mean salinity and temperature of the ArW, AtW or MIX, depending on which of these 3 water masses dominated at a station. At Stns C1 and C2, the mean salinity and temperature of the upper and lower depth strata were chosen. Ordination techniques and rules of interpretation of the ordination diagram have been reviewed by Jongman et al. (1995) and ter Braak \& Verdonschot (1995). In short, because of the relatively short gradient $(\sim 2 \mathrm{SD})$ in our species data, the position of species and samples can be determined using the biplot rule. The closer the species or samples cluster together, the more similar environmental preferences and species composition they have, respectively. Projection of species and samples perpendicular to the environmental variables (shown as arrows in Figs. $5 \& 6$ ) reveals their optima on these environmental gradients. Quantitative environmental variables are standardized and centred, and indicate the direction of maximum change. The angle between environmental arrows indicates their correlation, i.e. they are uncorrelated if they are perpendicular to each other and highly correlated (positive or negative) if the angle is small. Long arrows indicate higher correlation to the species patterns than shorter arrows.

Based on the results of CA and MDS, stations with similar species composition were grouped and total species abundance, biomass and richness were compared using non-parametric tests (Mann-Whitney $U$-test and Kruskal-Wallis median test; STATISCA 6.0).

\section{RESULTS}

\section{Ice conditions}

In May 1999, the ice edge followed the Polar Front in the study area (Fig. 1). The thaw had set in, and the ice receded markedly northwards during May. The ice was $<1.5 \mathrm{~m}$ thick and characterised as first-year ice. Transects A and B began in close pack-ice ( 7 to $9 / 10$ ), continued through open pack ice (3 to 6/10) and ended in open water $(1 / 10)$ along the ice edge (Table 1$)$. In March 2000, the ice edge in the central Barents Sea was located much further north of the Polar Front than in May 1999 (Fig. 1). Strong southerly winds pushed 
Fig. 1. Sampling stations and ice extent in the investigated area during May 1999 and March 2000. The Polar Front is shaded grey and is drawn thicker in areas where it is usually less defined (Loeng 1991). Atlantic and Arctic currents indicated by arrows

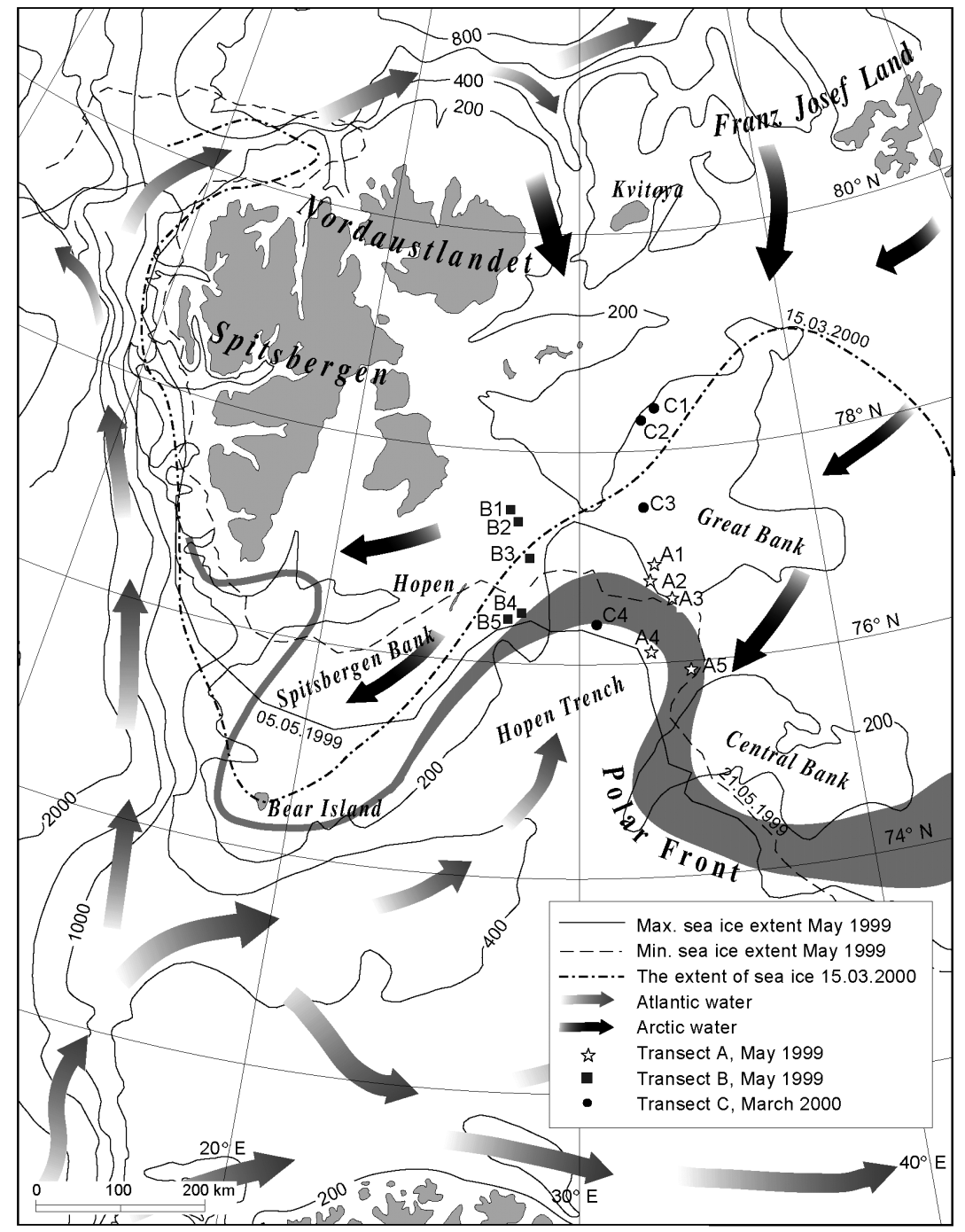

Table 1. Stations, cruise identity (ID) nos., dates, positions, bottom depths, volumes of filtered water (w.filt.) (mean \pm SE of 3 replicates), mean salinity and temperature of dominant water masses (Waterm.), ice concentrations (in tenths, NOAA 2001), ice ranking (1: open water, 2: open drift-ice, 3: close pack-ice), integrated ( 0 to $50 \mathrm{~m}$ ) chlorophyll a biomass and stage of algal bloom (0: non-bloom, 1: pre-bloom, 1.5 to 2.5: bloom, 3: late-bloom). ArW, AtW, MIX: Arctic, Atlantic and mixture of these water masses, respectively; nd: no data

\begin{tabular}{|c|c|c|c|c|c|c|c|c|c|c|c|c|}
\hline $\begin{array}{l}\text { Transect } \\
\text { station }\end{array}$ & $\begin{array}{l}\text { Cruise } \\
\text { ID }\end{array}$ & $\begin{array}{c}\text { Date } \\
\text { (d. mo. yr) }\end{array}$ & Position & $\begin{array}{l}\text { Depth } \\
\text { (m) }\end{array}$ & $\begin{array}{l}\text { w.filt. } \\
\text { (m) }\end{array}$ & Waterm. & $\begin{array}{c}\mathrm{S} \\
\text { (PSU) }\end{array}$ & $\begin{array}{c}T \\
\left({ }^{\circ} \mathrm{C}\right)\end{array}$ & $\begin{array}{l}\text { Ice } \\
\text { conc. }\end{array}$ & rank & $\begin{array}{r}\mathrm{Chl} \\
\left(\mathrm{mg} \mathrm{m}^{-2}\right)\end{array}$ & bloom \\
\hline A1 & A31 & 09.05 .99 & $76^{\circ} 55^{\prime} \mathrm{N}, 32^{\circ} 56^{\prime} \mathrm{E}$ & 160 & $302 \pm 2$ & ArW & 34.77 & -1.62 & $9 / 10$ & 3 & 162.1 & 1.5 \\
\hline A2 & A33 & 11.05 .99 & $76^{\circ} 48^{\prime} \mathrm{N}, 32^{\circ} 32^{\prime} \mathrm{E}$ & 186 & $344 \pm 2$ & ArW & 34.79 & -1.19 & $4-6 / 10$ & 2 & 286.6 & 2 \\
\hline A3 & A34 & 13.05 .99 & $76^{\circ} 38^{\prime} \mathrm{N}, 33^{\circ} 07^{\prime} \mathrm{E}$ & 159 & $323 \pm 2$ & ArW & 34.80 & -1.47 & $4-6 / 10$ & 2 & nd & 3 \\
\hline A4 & A35 & 14.05 .99 & $76^{\circ} 07^{\prime} \mathrm{N}, 32^{\circ} 20^{\prime} \mathrm{E}$ & 312 & $722 \pm 5$ & AtW & 35.01 & 1.56 & $1 / 10$ & 1 & 293.9 & 3 \\
\hline A5 & T15 & 07.05 .99 & $75^{\circ} 52^{\prime} \mathrm{N}, 34^{\circ} 25^{\prime} \mathrm{E}$ & 224 & $431 \pm 7$ & MIX & 34.98 & 1.03 & $1 / 10$ & 1 & 195.2 & 2.5 \\
\hline B1 & B49 & 17.05 .99 & $77^{\circ} 27^{\prime} \mathrm{N}, 27^{\circ} 00^{\prime} \mathrm{E}$ & 186 & $321 \pm 13$ & ArW & 34.44 & -1.41 & $7-9 / 10$ & 3 & 30.4 & 1 \\
\hline B2 & B50 & 18.05 .99 & $77^{\circ} 22^{\prime} \mathrm{N}, 27^{\circ} 10^{\prime} \mathrm{E}$ & 173 & $274 \pm 5$ & ArW & 34.44 & -1.37 & $7-9 / 10$ & 3 & 68.2 & 1 \\
\hline B3 & B51 & 20.05 .99 & $77^{\circ} 08^{\prime} \mathrm{N}, 27^{\circ} 57^{\prime} \mathrm{E}$ & 175 & $335 \pm 2$ & ArW & 34.57 & -1.22 & $4-6 / 10$ & 2 & 158.2 & 2.5 \\
\hline B4 & B52 & 21.05 .99 & $76^{\circ} 30^{\prime} \mathrm{N}, 27^{\circ} 43^{\prime} \mathrm{E}$ & 128 & $226 \pm 11$ & MIX & 34.92 & -0.05 & $1 / 10$ & 1 & 280.4 & 3 \\
\hline B5 & T3 & 05.05 .99 & $76^{\circ} 25^{\prime} \mathrm{N}, 27^{\circ} 07^{\prime} \mathrm{E}$ & 97 & $167 \pm 5$ & MIX & 34.87 & -0.82 & $1 / 10$ & 1 & nd & 2 \\
\hline C1 & C33 & 17.03 .00 & $78^{\circ} 21^{\prime} \mathrm{N}, 33^{\circ} 20^{\prime} \mathrm{E}$ & 179 & $304 \pm 2$ & ArW & 34.59 & -0.54 & $4-5 / 10$ & 2 & 0.1 & 0 \\
\hline $\mathrm{C} 2$ & C32 & 16.03 .00 & $78^{\circ} 16^{\prime} \mathrm{N}, 33^{\circ} 00^{\prime} \mathrm{E}$ & 156 & $326 \pm 38$ & ArW & 34.55 & -0.84 & $4 / 10$ & 2 & nd & 0 \\
\hline C3 & C35 & 18.03 .00 & $77^{\circ} 29^{\prime} \mathrm{N}, 32^{\circ} 51^{\prime} \mathrm{E}$ & 158 & $431 \pm 2$ & ArW & 34.61 & -1.55 & $4 / 10$ & 2 & nd & 0 \\
\hline $\mathrm{C} 4$ & C49 & 20.03 .00 & $76^{\circ} 30^{\prime} \mathrm{N}, 31^{\circ} 26^{\prime} \mathrm{E}$ & 317 & $832 \pm 57$ & AtW & 35.01 & 1.95 & 0 & 1 & 0.1 & 0 \\
\hline
\end{tabular}


the MIZ northwards, and large swells broke the ice into smaller floes $(<10 \mathrm{~m}$ in diameter). The ice was $<0.7 \mathrm{~m}$ thick, characterised as first-year ice. The first 3 stations (Stns C1 to C3) were in open pack-ice, whereas the last station (Stn C4) was in open water (0/10) (Table 1).

\section{Hydrography}

In May 1999, sampling was performed in the vicinity of the Polar Front and MIX was recorded at all stations (Figs. 1 \& 2). Cold ArW $\left(<-1.2^{\circ} \mathrm{C}\right)$ with salinities close to 34.8 PSU dominated over the slopes of the SW Great Bank (Stns A1 to A3), whereas warm, saline AtW (Stn A4) and less saline, but warm $\left(>1^{\circ} \mathrm{C}\right) \mathrm{MIX}$ (Stn A5) prevailed further south in the deeper Hopen Trench (Table 1). Cold ArW $\left(<-1.2^{\circ} \mathrm{C}\right)$ with salinities close to core ArW (34.3 to 34.5 PSU; Loeng 1991), covered by a surface melt-water layer (MW), dominated over the Spitsbergen Bank north of Hopen (Stns B1 to B3). In shallower areas, southeast of Hopen (Stns B4 and B5), cold MIX $\left(<0^{\circ} \mathrm{C}\right)$ prevailed. In March 2000, ArW dominated over the NW (Stns C1 and C2) and central Great Bank (Stn C3), whereas increased temperatures (0 to $0.5^{\circ} \mathrm{C}$ ) and salinities (34.6 to 34.8 PSU) occurred over the slopes of the NW Great Bank below 80 to $150 \mathrm{~m}$ depth (shown as an undefined water mass in Fig. 2). AtW dominated in the Hopen Trench (Stn C4).

\section{Pelagic algae}

In May 1999, the chlorophyll a biomass (0 to $50 \mathrm{~m}$ integrated) increased along both across-ice transects (A and B) from north to south, ranging from 162.1 to
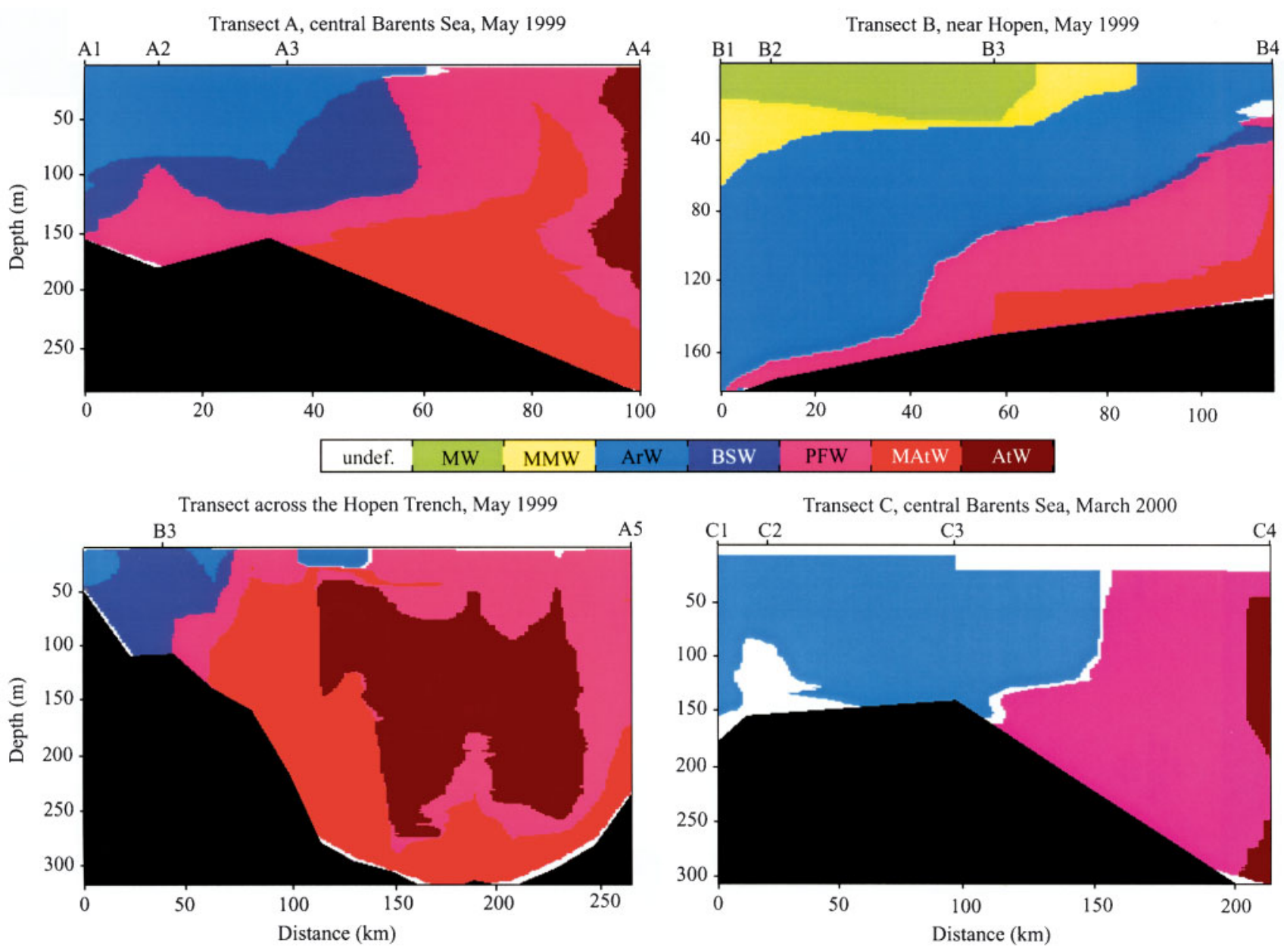

Fig. 2. Water masses along different sampling transects in May 1999 (Transects A and B) and March 2000 (Transect C). ArW: Arctic water $\left(34.3\right.$ to $\left.34.8 \mathrm{PSU},<0^{\circ} \mathrm{C}\right)$; AtW: Atlantic water ( $\left.>35.0 \mathrm{PSU},>1^{\circ} \mathrm{C}\right)$; MAtW: modified Atlantic water $(>34.95 \mathrm{PSU}$, -1.0 to $\left.1.0^{\circ} \mathrm{C}\right)$; PFW, BSW: warm and cold mixtures of AtW and $\mathrm{ArW}$, i.e. Polar Front water $\left(34.8\right.$ to $35.0 \mathrm{PSU}, 1.0$ to $3.0^{\circ} \mathrm{C}$; or 34.8 to $34.95 \mathrm{PSU},-0.5$ to $\left.1.0^{\circ} \mathrm{C}\right)$ and Barents Sea water $\left(34.8\right.$ to $\left.34.95 \mathrm{PSU},<-0.5^{\circ} \mathrm{C}\right)$, respectively; MW, MMW: melt water $(<34.2 \mathrm{PSU})$ and mixed melt water $\left(34.2\right.$ to $\left.34.3 \mathrm{PSU},<0^{\circ} \mathrm{C}\right)$ introduced through melting of sea ice, respectively; undef.: undefined water masses. Note differences in ordinate and abscissa scales 
$293.9 \mathrm{mg} \mathrm{m}^{-2}$ and 30.4 to $280.4 \mathrm{mg} \mathrm{m}^{-2}$ at Transects A and $\mathrm{B}$, respectively (Table 1). Algae in bloom and latebloom phase prevailed along Transect A, whereas the algal bloom had barely started (pre-bloom) at the 2 northernmost stations (B1 and B2) of Transect B (Table 1). However, in less dense ice further south on Transect B (Stns B3 and B4), the algal bloom had progressed for some time and had reached late-bloom phase (deep chlorophyll a maximum). In March 2000, the phytoplankton biomass (0 to $50 \mathrm{~m}$ integrated) showed typically low winter values $\left(<0.1 \mathrm{mg} \mathrm{chl} \mathrm{a} \mathrm{m}^{-2}\right)$ and no algal blooms were recorded (Table 1) (E. N. Hegseth pers. comm.).

\section{Macrozooplankton}

A total of 27 macrozooplankton taxa, including 20 taxa at species level, were recorded during May 1999 and March 2000, of which 18 species and 2 taxa were found on both cruises (Table 2). The only additional species sampled in March was the pteropod Limacina helicina, but its abundance was very low $1<0.2$ individuals [ind.] $\mathrm{m}^{-2}$ ). Half the recorded species were found at $>70 \%$ of the stations, but their abundance at the different stations varied (Table 2); 15 species and 1 taxon were mainly carnivorous, 2 species were mainly omnivorous, and 3 species were mainly herbivorous (Table 2). The feeding category of the remaining 6 taxa (which were rarely found) was undetermined.

Hydromedusae (Aglantha digitale), ctenophores (Beröe cucumis and Mertensia ovum), pteropods (Clione limacina), euphausiids (Thysanoessa longicaudata and T. inermis) and chaetognaths (Eukrohnia hamata and Sagitta elegans) were relatively abundant and divided into small (S), medium (M) and large (L) individuals (Table 2, Fig. 3). Other relatively abundant species were the hydromedusae Aeginopsis laurentii and Sarsia spp. (sizes not measured) and the siphonophore Dimophyes arctica. The hyperiid amphipods Parathemisto abyssorum (median TL $=10 \mathrm{~mm})$, $P$. libellula (median TL $=$ $22 \mathrm{~mm}$ ), and Hyperia galba (median TL = $6 \mathrm{~mm}$ ) and the remaining non-copepod taxa were found in too low abundances to be divided into size classes. The abundant copepods Calanus glacialis and C. hyperboreus and the less abundant Pareuchaeta spp. were divided into developmental stages.

The life histories of Aglantha digitale, Beröe cucumis, Mertensia ovum, Clione limacina, Eukhrania hamata and Sagitta elegans in the Barents Sea are largely unknown. Since the smallest individuals were poorly sampled (Fig. 3), possible cohorts could not be estimated from their size distributions alone. They were therefore divided into size groups (small, medium and large) of regular lengths (Table 2).
The size distribution of Thysanoessa longicaudata and $T$. inermis in the present study consisted of at least 3 cohorts (year classes), determined according to Dalpadado \& Skjoldal (1996) (Fig. 3). Small T. inermis (<16 mm) (juveniles only) represented the 0 and 1 st cohorts, whereas medium (16 to $22 \mathrm{~mm}$ ) and large specimens (>22 $\mathrm{mm}$ ) (all mature) represented the 2nd and the 3rd cohort. Small T. longicaudata $(<12 \mathrm{~mm})$ (mix of juvenile and mature) made up the 0 and 1 st cohorts, whereas medium (12 to $15 \mathrm{~mm}$ ) and large (>15 mm) T. longicaudata (all mature) constituted the 2nd and the 3rd cohorts.

\section{Sample replicability}

The 3 replicates at each station showed high similarity in species abundance (60 to $80 \%$ similarity, SIMPER), except for the 3 replicates at Stn C4, sampled in AtW in March 2000 (Fig. 4a). These 3 replicates varied greatly in total abundance (8.1, 19.9 and 99.4 ind. $\mathrm{m}^{-2}$ ), and showed only $38 \%$ similarity in species abundance (SIMPER). The sample variability within stations was less than between stations (except for Stn C4), and stations located close to each other, with similar hydrographical characteristics (i.e. samples obtained from Stns A1-A3, B1-B3 and C1-C2), showed high similarity in species abundance $(80,69$ and $66 \%$ similarity, respectively). A gradual change in the macrozooplankton composition from AtW to ArW existed, in terms of both species abundance and biomass (Fig. 4). Stations dominated by MIX (Stns A5, B4 and B5) differed from stations dominated by either ArW or AtW, mainly because of their low total abundance $\left(<35\right.$ ind. $\left.\mathrm{m}^{-2}\right)$ and biomass $\left(<1.2 \mathrm{~g} \mathrm{~m}^{-2}\right)$. A gradual change in species abundance and biomass was also found from warm (Stn A5) to cold MIX (Stns B4 and B5) stations.

In terms of species biomass, the replicates were 48 to $73 \%$ similar, except at Stn $\mathrm{C} 4$, where the 3 replicates were only $13 \%$ similar in species biomass (SIMPER).

\section{Environmental influence on species patterns (CA and CCA)}

The macrozooplankton distribution in the MIZ in the Barents Sea was significantly correlated to the measured environmental variables latitude, longitude, salinity, temperature, bottom depth and season (Table 1), which together explained almost $80 \%$ of the total macrozooplankton variability during May 1999 and March 2000 (Table 3, Fig. 5). The ice concentration and algal bloom stage (Table 1) had no additional impact $(\mathrm{p}=0.1$ and 0.8 , respectively) on the 
Table 2. Macrozooplankton species in the Barents Sea marginal ice zone in May 1999 (spring) and March 2000 (late winter), showing frequency of occurrence (Freq.) and mean $( \pm \mathrm{SE})$, minimum and maximum abundance at the 14 stations (total of 42 samples) sampled (f: female; m: male; indet.: indeterminate). Station with maximum abundance (Stn max.) is listed for each taxon. For correspondence analysis (CA) the percentage of species variability explained by environmental variables in 2-dimensional ordination plots (Expl. 2-dim.) is listed together with taxon abbreviations (Abbr.) used in Figs. 5 \& 6. Taxa grouped by feeding mode (h: herbivore; o: omnivore; c: carnivore; u: unknown) and zoogeographical (zoogeo.) distribution (a: Arctic; ab: Arctic-boreal; ba: boreal-Arctic; c: cosmopolitan; u: unknown) from data derived from literature (sources)

\begin{tabular}{|c|c|c|c|c|c|c|c|c|c|c|}
\hline \multicolumn{2}{|l|}{ Taxon (feeding mode) } & Abbr. & \multirow{2}{*}{$\begin{array}{l}\text { Freq. } \\
(\%)\end{array}$} & \multicolumn{2}{|c|}{ Abundance (ind. $\mathrm{m}^{-2}$ ) } & \multirow{2}{*}{$\begin{array}{l}\text { Stn } \\
\text { max. }\end{array}$} & \multicolumn{2}{|c|}{ Expl. 2-dim. (\%) } & Zoogeo & Source \\
\hline \multicolumn{9}{|l|}{ Hydromedusa } & & \\
\hline Aglantha digitale (O.F. Müller) & (c) & & $93^{+}$ & $7.3 \pm 0.7$ & $0.1-31.2$ & $\mathrm{C} 1$ & & & $\mathrm{ab}$ & Rasmussen (1971), Zelikman (1972) \\
\hline A. digitale $<10 \mathrm{~mm}$ & (c) & Ad S & $71^{+}$ & $2.0 \pm 0.7$ & $0.2-7.9$ & A3 & 7.3 & 74.1 & $\mathrm{ab}$ & \\
\hline A. digitale $10-15 \mathrm{~mm}$ & (c) & Ad M & $86^{+}$ & $3.8 \pm 1.1$ & $0.2-13.3$ & $\mathrm{C} 1$ & 55.2 & 73.1 & $\mathrm{ab}$ & \\
\hline A. digitale $>15 \mathrm{~mm}$ & (c) & Ad L & $79^{+}$ & $1.4 \pm 0.4$ & $0.2-3.8$ & A4 & 51.4 & 72.6 & $\mathrm{ab}$ & \\
\hline Aeginopsis laurentii Brandt & (c) & Alau & $71^{+}$ & $2.1 \pm 1.4$ & $0.2-18.4$ & $\mathrm{C} 1$ & 64.1 & 36.4 & $\mathrm{ab}$ & Zelikman (1972), Madin (1988) \\
\hline Sarsia spp. & (c) & Sar & $71^{+}$ & $0.8 \pm 0.3$ & $0.1-4.4$ & A3 & 41.5 & 30.3 & $\mathrm{u}$ & Madin (1988) \\
\hline \multicolumn{11}{|l|}{ Siphonophora } \\
\hline Dimophyes arctica (Chun) & (c) & Darc & $57^{+}$ & $3.6 \pm 2.5$ & $0.1-34.0$ & $\mathrm{C} 1$ & 75.6 & 15.4 & C & Zelikman (1972), Madin (1988) \\
\hline Siphonophora indet. & (u) & $\operatorname{Siph}^{*}$ & 7 & $0.1 \pm 0.1$ & 1.4 & A4 & 26.5 & 27.2 & $\mathrm{u}$ & \\
\hline \multicolumn{11}{|l|}{ Ctenophora } \\
\hline Beröe cucumis Fabricius & (c) & & $100^{+}$ & $5.1 \pm 0.8$ & $0.9-11.5$ & B1 & & & $\mathrm{ab}$ & $\begin{array}{l}\text { Zelikman (1972), } \\
\text { Falk-Petersen et al. (2002) }\end{array}$ \\
\hline B. cucumis $<20 \mathrm{~mm}$ & (c) & Bcu S & $100^{+}$ & $3.8 \pm 0.8$ & $0.7-11.0$ & B1 & 51.7 & 6.7 & $\mathrm{ab}$ & \\
\hline B. cucumis $20-39 \mathrm{~mm}$ & (c) & ' & $93^{+}$ & $1.1 \pm 0.3$ & $0.1-3.6$ & $\mathrm{C} 1$ & 0.5 & 3.6 & $\mathrm{ab}$ & \\
\hline B. cucumis $>39 \mathrm{~mm}$ & (c) & Bcu L & $86^{+}$ & $0.3 \pm 0.1$ & $0.1-0.7$ & A1 & 26.3 & 6.7 & $\mathrm{ab}$ & \\
\hline Mertensia ovum (Fabricius) & (c) & & $64^{+}$ & $1.0 \pm 0.4$ & $0.3-4.2$ & B1 & & & a & $\begin{array}{l}\text { Ekman (1953), } \\
\text { Falk-Petersen et al. (2002) }\end{array}$ \\
\hline M. ovum $<20 \mathrm{~mm}$ & (c) & Mo S & $50^{+}$ & $0.4 \pm 0.2$ & $0.2-2.5$ & B2 & 69.6 & 57.4 & a & \\
\hline M. ovum $20-39 \mathrm{~mm}$ & (c) & Mo M & $64^{+}$ & $0.4 \pm 0.2$ & $0.1-1.5$ & B2 & 78.8 & 67.8 & $\mathrm{a}$ & \\
\hline M. ovum > $39 \mathrm{~mm}$ & (c) & Mo L & $36^{+}$ & $0.2 \pm 0.1$ & $0.2-0.8$ & B2 & 71.7 & 66.8 & a & \\
\hline \multicolumn{11}{|l|}{ Sipuncula } \\
\hline Sipuncula indet. & (u) & Sipun* & 7 & $0.0 \pm 0.0$ & 0.1 & A4 & 26.5 & 27.2 & $\mathrm{u}$ & \\
\hline \multicolumn{11}{|l|}{ Pteropoda } \\
\hline Clione limacina Phipps & (c) & & $93^{+}$ & $1.3 \pm 0.4$ & $0.2-4.4$ & B2 & & & $\mathrm{ab}$ & Colin \& Satterlie (1992) \\
\hline C. limacina $<20 \mathrm{~mm}$ & (c) & Cli S & $71^{+}$ & $0.7 \pm 0.2$ & $0.2-3.0$ & B2 & 76.6 & 60.8 & $\mathrm{ab}$ & \\
\hline C. limacina 20-39 mm & (c) & Cli M & $86^{+}$ & $0.4 \pm 0.1$ & $0.1-1.2$ & B2 & 74 & 72.6 & $\mathrm{ab}$ & \\
\hline C. limacina $>39 \mathrm{~mm}$ & (c) & Cli L & $57^{+}$ & $0.2 \pm 0.0$ & $0.1-0.5$ & $\mathrm{C} 2$ & 33.8 & 48.2 & $\mathrm{ab}$ & \\
\hline Limacina helicina Phipps & (o) & Lhe* & 7 & $0.0 \pm 0.0$ & 0.2 & $\mathrm{C} 1$ & 42.3 & 8.6 & $\mathrm{ab}$ & Colin \& Satterlie (1992) \\
\hline \multicolumn{11}{|l|}{ Copepoda } \\
\hline Calanus glacialis Jaschnov CVI & (h) & & $100^{+}$ & $64.5 \pm 22.5$ & $1.2-292.3$ & B2 & & & a & Eilertsen et al. (1989) \\
\hline C. glacialis CVI f & (h) & CgVIf & $100^{+}$ & $63.8 \pm 22.3$ & $1.2-290.6$ & B2 & 72. & 70.4 & a & \\
\hline C. glacialis CVI m & (h) & CgVIm & $64^{+}$ & $0.7 \pm 0.3$ & $0.2-3.7$ & $\mathrm{C} 1$ & 50.1 & 43.4 & a & \\
\hline Calanus hyperboreus Krøyer CV-CVI & (h) & & $100^{+}$ & $21.2 \pm 4.0$ & $2.9-48.1$ & A4 & & & a & Eilertsen et al. (1989) \\
\hline C. hyperboreus CVI f & (h) & ' & $100^{+}$ & $9.6 \pm 2.0$ & $1.3-27.0$ & $\mathrm{C} 2$ & 7.4 & 7.4 & $\mathrm{a}$ & \\
\hline C. hyperboreus CVI m & (h) & ChVIm & $34^{+}$ & $0.7 \pm 0.5$ & $0.1-5.5$ & $\mathrm{C} 1$ & 79.8 & 3 & a & \\
\hline C. hyperboreus CV & (h) & $\mathrm{ChV}$ & $100^{+}$ & $8.8 \pm 2.6$ & $0.4-35.0$ & A4 & 61 & 2,2 & a & \\
\hline C. hyperboreus CIV & (h) & ChIV & $71^{+}$ & $2.1 \pm 1.0$ & $0.3-10.3$ & A2 & 6.9 & 46 & a & \\
\hline Pareuchaeta spp. CIV-CVI & (c) & & $93^{+}$ & $3.6 \pm 1.3$ & $0.2-15.9$ & $\mathrm{C} 1$ & & & a & \\
\hline P. norvegica (Boeck) CVI f & (c) & PnVlf & $79^{+}$ & $0.7 \pm 0.3$ & $0.1-2.7$ & $\mathrm{C} 1$ & 51.3 & 32.7 & $\mathrm{ab}$ & $\begin{array}{l}\text { Harding (1974), } \\
\text { Klekowski \& Weslawski (1991) }\end{array}$ \\
\hline P. glacialis (Hansen) CVI f & (c) & PgVIf & $21^{+}$ & $0.1 \pm 0.0$ & $0.2-0.5$ & A1 & 11.4 & 30.8 & a & $\begin{array}{l}\text { Longhurst (1976), } \\
\text { Klekowski \& Weslawski (1991) }\end{array}$ \\
\hline Pareuchaeta spp. CVI m & (c) & ParVIm & $29^{+}$ & $0.1 \pm 0.1$ & $0.4-0.8$ & $\mathrm{C} 1$ & 80.1 & 20.8 & a & \\
\hline Pareuchaeta spp. CV & (c) & ParV & $86^{+}$ & $2.5 \pm 1.0$ & $0.2-11.2$ & $\mathrm{C} 1$ & 32.1 & 13 & a & \\
\hline Pareuchaeta spp. CIV & (c) & ParlV & $21^{+}$ & $0.1 \pm 0.1$ & $0.2-1.0$ & $\mathrm{C} 1$ & 79.1 & 4.4 & a & \\
\hline \multicolumn{11}{|l|}{ Mysidacea } \\
\hline Mysidacea indet. & (u) & Mys* $^{*}$ & 7 & $0.0 \pm 0.0$ & 0.7 & A5 & 29.7 & 32.7 & $\mathrm{u}$ & \\
\hline
\end{tabular}


Table 2 (continued)

\begin{tabular}{|c|c|c|c|c|c|c|c|c|c|c|}
\hline \multicolumn{2}{|l|}{ Taxon (feeding mode) } & \multirow[t]{2}{*}{ Abbr. } & \multirow[t]{2}{*}{$\begin{array}{l}\text { Freq. } \\
(\%)\end{array}$} & \multirow[t]{2}{*}{$\begin{array}{l}\text { Abundanc } \\
\text { Mean } \pm \text { SE }\end{array}$} & \multirow[t]{2}{*}{$\begin{array}{l}\text { (ind. } \mathrm{m}^{-2} \text { ) } \\
\text { min.-max. }\end{array}$} & \multirow[t]{2}{*}{$\begin{array}{l}\text { Stn } \\
\text { max. }\end{array}$} & \multicolumn{2}{|c|}{ Expl. 2-dim. (\%) } & Zoogeo & Source \\
\hline \multicolumn{6}{|l|}{ Amphipoda } & & & & & \\
\hline Parathemisto abyssorum Boeck & (c) & $\mathrm{Pab}$ & $21^{+}$ & $0.1 \pm 0.0$ & $0.2-0.4$ & B3 & 15.0 & 15.8 & $\mathrm{ab} \quad \mathrm{I}$ & $\begin{array}{l}\text { Dunbar (1954), } \\
\text { Klekowski \& Weslawski (1991) }\end{array}$ \\
\hline Parathemisto libellula (Boeck) & (c) & Pli & $57^{+}$ & $0.4 \pm 0.1$ & $0.2-2.1$ & B2 & 56.8 & 60.2 & a & $\begin{array}{l}\text { Dunbar (1964), } \\
\text { Klekowski \& Weslawski (1991) }\end{array}$ \\
\hline Hyperia galba (Montagu) & (c) & Hga & $43^{+}$ & $0.6 \pm 0.3$ & $0.3-3.3$ & $\mathrm{C} 1$ & 75.4 & 8.8 & $\begin{array}{lll}\mathrm{ab} & 1 \\
& \mathrm{I}\end{array}$ & $\begin{array}{l}\text { Klekowski \& Weslawski (1991), } \\
\text { Dittrich (1992) }\end{array}$ \\
\hline Onisimus spp. & (u) & Oni & $14^{+}$ & $0.0 \pm 0.0$ & $0.1-0.2$ & $\mathrm{C} 2$ & 23.1 & 11 & $\mathrm{u}$ & \\
\hline \multicolumn{11}{|l|}{ Euphausiacea } \\
\hline Thysanoessa longicaudata (Krøyer) & (c) & & $93^{+}$ & $1.8 \pm 0.5$ & $0.2-7.3$ & A4 & & & ba $\frac{1}{1}$ & $\begin{array}{l}\text { Klekowski \& Weslawski (1991), } \\
\text { Falk-Petersen et al. (2000b) }\end{array}$ \\
\hline T. longicaudata $<12 \mathrm{~mm}$ & (c) & Tlo S & $71^{+}$ & $1.3 \pm 0.5$ & $0.2-6.3$ & A4 & 70.2 & 71.7 & ba & \\
\hline T. longicaudata $12-15 \mathrm{~mm}$ & (c) & Tlo M & $86^{+}$ & $0.5 \pm 0.2$ & $0.2-2.2$ & $\mathrm{C} 2$ & 18.9 & 29.9 & ba & \\
\hline T. longicaudata $>15 \mathrm{~mm}$ & (c) & Tlo L & 14 & $0.0 \pm 0.0$ & $0.1-0.4$ & $\mathrm{C} 2$ & 24.6 & 4.9 & ba & \\
\hline Thysanoessa inermis (Krøyer) & (h) & & $93^{+}$ & $6.0 \pm 2.2$ & $0.6-30.0$ & A4 & & & ba $\frac{1}{1}$ & $\begin{array}{l}\text { Klekowski \& Weslawski (1991), } \\
\text { Falk-Petersen et al. (2000b) }\end{array}$ \\
\hline T. inermis $<16 \mathrm{~mm}$ & (h) & Ti S & $86^{+}$ & $3.9 \pm 1.3$ & $0.2-16.8$ & A4 & 85.4 & 72.8 & ba & \\
\hline T. inermis $16-22 \mathrm{~mm}$ & (h) & Ti M & $79^{+}$ & $1.3 \pm 0.6$ & $0.5-9.0$ & A4 & 38.6 & 53.6 & ba & \\
\hline T. inermis $>22 \mathrm{~mm}$ & (h) & Ti L & 29.0 & $0.7 \pm 0.4$ & $0.2-4.7$ & A5 & 59.2 & 64.4 & ba & \\
\hline Thysanoessa raschii (M. Sars) & (o) & Tra & $64^{+}$ & $0.3 \pm 0.1$ & $0.2-0.9$ & $\mathrm{C} 2$ & 41.7 & 49.7 & ba 1 & $\begin{array}{l}\text { Klekowski \& Weslawski (1991), } \\
\text { Falk-Petersen et al. (2000b) }\end{array}$ \\
\hline Meganyctiphanes norvegica (M. Sars) & (c) & $*, /$ & 7 & $0.0 \pm 0.0$ & 0.5 & B3 & 9.3 & 12.1 & $\mathrm{~b}$ & $\begin{array}{l}\text { Klekowski \& Weslawski (1991), } \\
\text { Falk-Petersen et al. (2000b) }\end{array}$ \\
\hline \multicolumn{11}{|l|}{ Decapoda } \\
\hline Decapoda larvae & (u) & Deca & 50 & $0.3 \pm 0.1$ & $0.1-1.4$ & A5 & 28.1 & 11.1 & $\mathrm{u}$ & \\
\hline \multicolumn{11}{|l|}{ Chaetognatha } \\
\hline Eukrohnia hamata (Mobius) & (c) & & $79^{+}$ & $2.9 \pm 1.2$ & $0.1-14.9$ & $\mathrm{C} 1$ & & & $\mathrm{C}$ & Pierrot-Bults \& Chidgey (1988) \\
\hline E. hamata $<19 \mathrm{~mm}$ & (c) & Eha S & $43^{+}$ & $0.4 \pm 0.2$ & $0.2-2.1$ & $\mathrm{C} 2$ & 64.9 & 7.3 & $\mathrm{C}$ & \\
\hline E. hamata $20-28 \mathrm{~mm}$ & (c) & Eha M & $71^{+}$ & $1.7 \pm 0.6$ & $0.2-7.5$ & $\mathrm{C} 1$ & 65.1 & 58.7 & $\mathrm{C}$ & \\
\hline E. hamata $>28 \mathrm{~mm}$ & (c) & Eha L & $64^{+}$ & $0.4 \pm 0.2$ & $0.1-3.5$ & $\mathrm{C} 1$ & 51.3 & 21.9 & c & \\
\hline Sagitta elegans arctica Aurivillius & (c) & & $93^{+}$ & $3.3 \pm 2.2$ & $0.5-10.0$ & $\mathrm{C} 1$ & & & $\mathrm{ab} \quad \mathrm{l}$ & Pierrot-Bults \& Chidgey (1988) \\
\hline S. elegans $<29 \mathrm{~mm}$ & (c) & Sel S & $57^{+}$ & $0.6 \pm 0.2$ & $0.2-2.2$ & $\mathrm{C} 4$ & 3.5 & 25.7 & $\mathrm{ab}$ & \\
\hline S. elegans $29-36 \mathrm{~mm}$ & (c) & Sel M & $93^{+}$ & $2.3 \pm 0.7$ & $0.2-8.0$ & $\mathrm{C} 1$ & 17.1 & 30.8 & $\mathrm{ab}$ & \\
\hline S. elegans $>36 \mathrm{~mm}$ & (c) & Sel L & $57^{+}$ & $0.4 \pm 0.2$ & $0.2-1.4$ & $\mathrm{C} 3$ & 34.7 & 51 & $\mathrm{ab}$ & \\
\hline \multicolumn{11}{|l|}{ Vertebrata } \\
\hline Pisces larvae & (u) & Pisces & 29 & $0.1 \pm 0.0$ & $0.2-0.4$ & B2 & 78 & 52.1 & $\mathrm{u}$ & \\
\hline
\end{tabular}

macrozooplankton patterns after salinity and temperature (which were highly correlated with the fraction of ice concentration; $r>-0.83$ ) and season (which was highly correlated to bloom stage; $r=0.84$ ) were taken into account. There was a high degree of faunal similarity among the samples, which was reflected by the relatively low eigenvalues $(<0.22)$ and low total inertia $(<0.8)$ (Table 4). Normally, eigenvalues of 0.3 to 0.5 indicate good separation of species or samples, i.e. that stations have few species in common (ter Braak \& Verdonschot 1995).
There were 2 main macrozooplankton gradients, one along the salinity and temperature gradients and one along the seasonal (March to May) gradient, which was not correlated with the salinity $(\mathrm{r}=0.22)$ or temperature ( $\mathrm{r}=-0.16$ ) gradients (Fig. 5). Geographical position was correlated to salinity, temperature and season, and latitude was more highly correlated with these 3 environmental variables $(r=-0.80,-0.47$ and -0.67 , respectively) than longitude $(r=0.38,0.20$ and -0.42 , respectively). Geographical position (latitude and longitude), which explained a significant 
additional part of species variability, explained a slightly higher percentage $(33 \%)$ of the macrozooplankton variability than did water masses (31\%) (Table 3). However, after subtracting seasonal variability, which accounted for $21 \%$ of total macrozooplankton variability, the water masses explained almost twice as much of the species variability than geographical position (40 vs $25 \%$ ). The macrozooplankton composition over the slopes of the NW (Stns C1 and C2) and SW (Stns A1 to A3) Great Bank displayed high similarity after adjusting for seasonal macrozooplankton variability; thus, 1 strong macrozooplankton gradient, highly correlated with salinity gradient, appeared (Table 3, Fig. 6).

\section{Species and their environmental optima}

The environmental preferences for macrozooplankton, defined as Arctic and boreal-Arctic taxa in the literature (Table 2), corresponded well with the environmental variables in the ordination plots (Figs. 5b \&
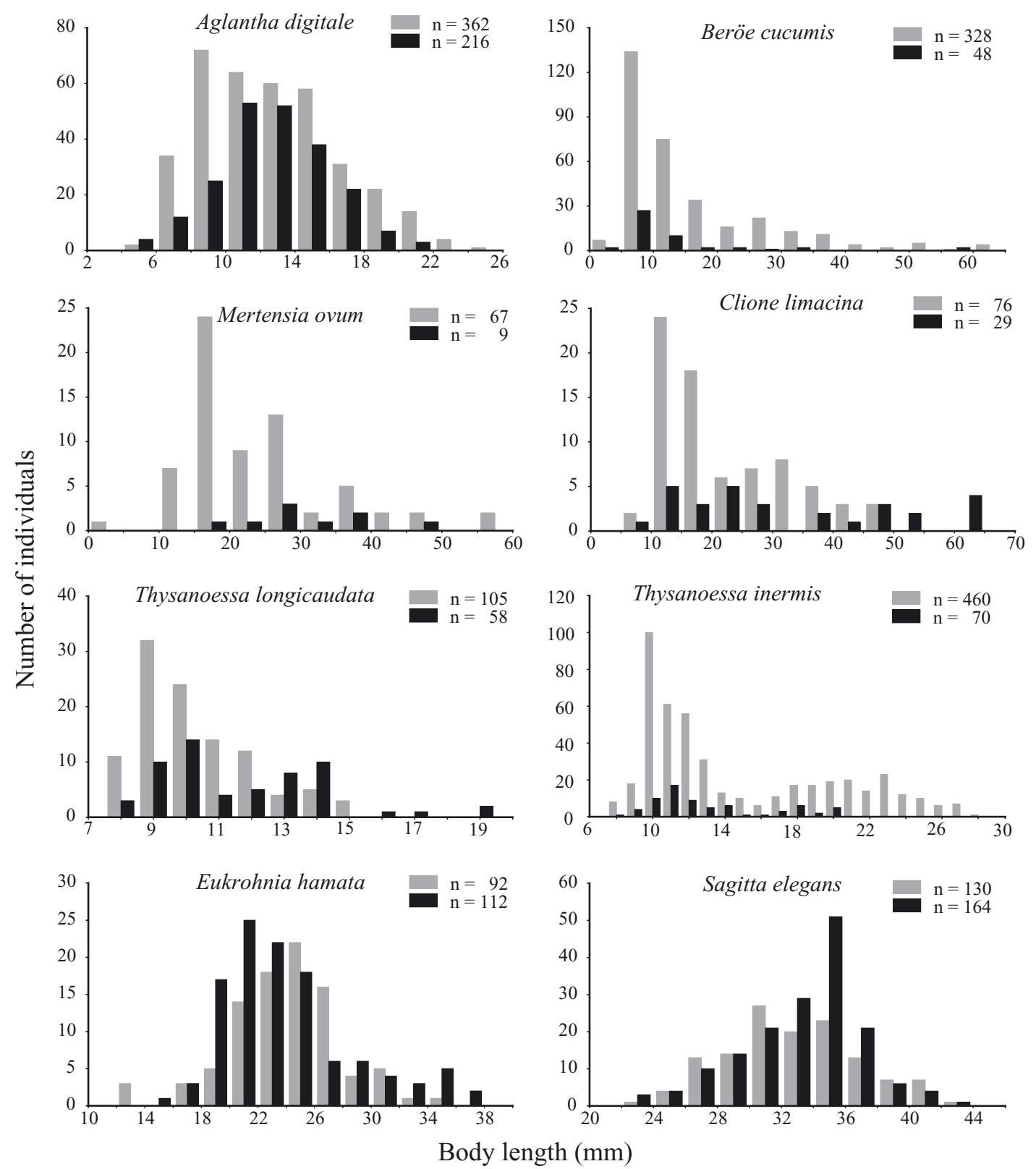

Fig. 3. Length-frequency histograms of selected macrozooplankton during May 1999 (grey columns) and March 2000 (black columns) in the Barents Sea marginal ice zone. Note differences in ordinate and abscissa scales 
6b). Arctic taxa were concentrated in cold and less saline waters over the banks (Stns B1 to B3 and C3), whereas boreal-Arctic taxa were concentrated in warm and saline waters in the deeper Hopen Trench (Stns A4, A5 and C4). Arctic-boreal taxa were scattered, but after seasonal variability was removed they were confined to ArW either over the banks (ArW bank, Stns B1 to B3 and Stn C3) or over the slopes (ArW slope, Stns A1 to A3 and Stns C1 and C2). The cosmopolitan species Dimophyes arctica and Eukrohnia hamata showed high seasonality in their abundance, which peaked during March on the ArW slope (Stns C1 and C2).
After seasonal variability has been removed, small, medium and large individuals of various species displayed relatively similar environmental preferences (except for Sagitta elegans), although medium-sized specimens were generally more widely distributed than the small and large specimens (Figs. 5b \& 6b). Prior to dividing species into developmental stages (copepods) and size classes (abundant non-copepod species), seasonal macrozooplankton variability was non-significant $(p=0.07)$. Even though there was less heterogeneity in species data prior to division into developmental stages and size classes, a higher percentage of the data was explained by environmental

a)

Abundance (ind. $\mathrm{m}^{-2}$ )

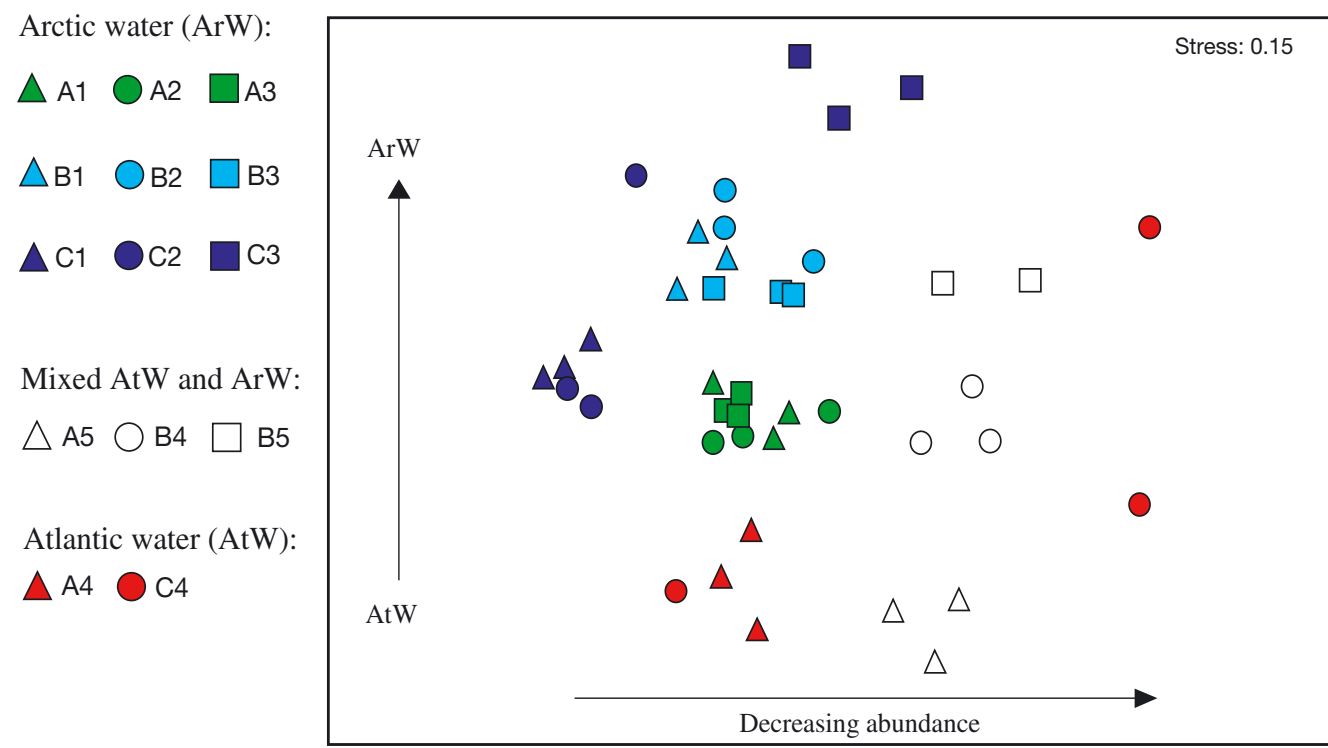

b)

Wet weight $\left(\mathrm{g} \mathrm{m}^{-2}\right)$

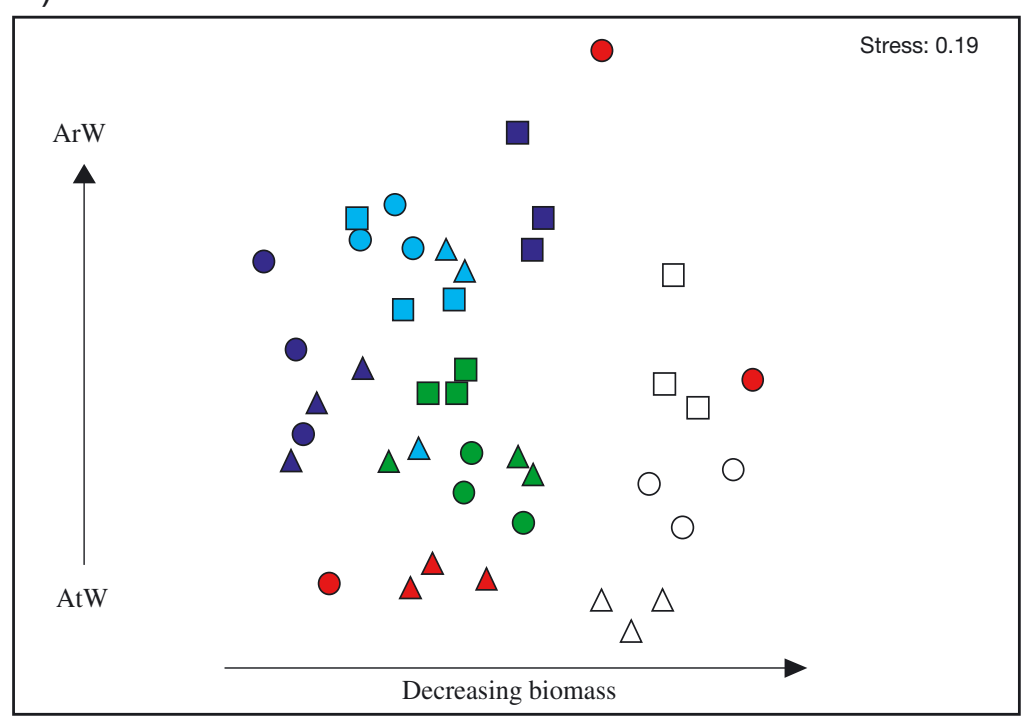

Fig. 4. MDS-plot of (a) species abundance (indivuals $\mathrm{m}^{-2}$ ) and (b) species biomass for 42 samples collected in the Barents Sea marginal ice zone during May 1999 (Stns A and B) and March 2000 (Stn C), based on Bray-Curtis similarity. For the abundance analysis (a), copepods were divided into developmental stages and abundant non-copepods into size classes, but not for the wet biomass analysis (b). Water abbreviations as in Fig. 2 
Table 3. Ranking of environmental variables that significantly (Monte Carlo permutation test in CCA, p < 0.05) influenced distribution of macrozooplankton in the Barents Sea marginal ice zone in May 1999 (spring) and March 2000 (late winter). Environmental variable that best explains macrozooplankton distribution is ranked first, remaining variables are ranked on basis of additional fit. (A) Seasonal variability included; (B) seasonal variability removed

\begin{tabular}{|c|c|c|c|c|c|c|}
\hline \multirow{2}{*}{$\begin{array}{l}\text { Environmental } \\
\text { variable }\end{array}$} & \multirow{2}{*}{$\begin{array}{c}\mathrm{p}- \\
\text { value }\end{array}$} & \multirow{2}{*}{$\begin{array}{c}F- \\
\text { value }\end{array}$} & \multicolumn{2}{|c|}{ Explained } & \multicolumn{2}{|c|}{ Correlation } \\
\hline & & & Inertia & $\%$ & Axis 1 & Axis 2 \\
\hline \multicolumn{7}{|l|}{ (A) Season incl. } \\
\hline Latitude $\left({ }^{\circ} \mathrm{N}\right)$ & 0.001 & 4.33 & 0.20 & 26.1 & -0.96 & -0.11 \\
\hline Salinity (PSU) & 0.001 & 4.46 & 0.17 & 22.2 & 0.81 & -0.46 \\
\hline Temperature $\left({ }^{\circ} \mathrm{C}\right)$ & 0.004 & 2.45 & 0.07 & 9.1 & 0.55 & -0.58 \\
\hline Depth (m) & 0.035 & 1.85 & 0.06 & 7.8 & 0.46 & -0.40 \\
\hline Longitude $\left({ }^{\circ} \mathrm{E}\right)$ & 0.018 & 2.12 & 0.05 & 6.5 & 0.02 & -0.76 \\
\hline Season (March/May) & 0.030 & 2.34 & 0.06 & 7.8 & 0.63 & 0.57 \\
\hline Total & 0.001 & 4.49 & 0.61 & 79.6 & & \\
\hline \multicolumn{7}{|l|}{ (B) Season excl. } \\
\hline Salinity (PSU) & 0.001 & 4.53 & 0.18 & 29.8 & 0.92 & 0.02 \\
\hline Latitude $\left({ }^{\circ} \mathrm{N}\right)$ & 0.003 & 2.79 & 0.09 & 14.9 & -0.81 & -0.37 \\
\hline Temperature $\left({ }^{\circ} \mathrm{C}\right)$ & 0.016 & 2.12 & 0.06 & 9.9 & 0.84 & 0.32 \\
\hline Depth (m) & 0.034 & 2.02 & 0.06 & 9.9 & 0.63 & 0.22 \\
\hline Longitude $\left({ }^{\circ} \mathrm{E}\right)$ & 0.032 & 2.57 & 0.06 & 9.9 & 0.61 & -0.40 \\
\hline Total & 0.001 & 3.95 & 0.45 & 74.5 & & \\
\hline
\end{tabular}

markedly reduced (Table 2 , Figs. 5b \& 6b). After adjusting for seasonal variability, 3 major centres of macrozooplankton distribution were found: (1) in cold and less saline waters over the banks (Aeginopsis laurentii, Sarsia spp., Mertensia ovum, Clione limacina, Calanus glacialis, Parathemisto libellula and Sagitta elegans $>29 \mathrm{~mm}$ ); (2) in cold, but relatively saline waters over the slopes (Aglantha digitale, D. arctica, Pareuchaeta spp. CVI and E. hamata $\geq 20 \mathrm{~mm}$ ); and (3) in warm and saline waters in the deeper Hopen Trench (Thysanoessa longicaudata $\leq 15 \mathrm{~mm}, T$. inermis and $T$. raschii and the rarely occurring taxa Siphonophora indet., Sipuncula indet. and Mysidacea indet.). Medium-sized B. cucumis and females of $C$. hyperboreus showed no consistent gradient in species abundance, and had a poor fit ( $<10 \%$ variability explained) in the CA (Table 2).

\section{Abundance, biomass and species richness}

Stations with similar relative macrozoovariables after such division (74.4 vs $79.4 \%$ ). In addition, more information on the macrozooplankton community structure was gained.

After subtracting seasonal macrozooplankton variability, the species-environment correlations of macrozooplankton concentrated along the seasonal gradient (i.e. Dimophyes arctica, small and large Beröe cucumis, Calanus hyperboreus CV, Pareuchaeta spp. CV-CVI [male] and small and large Eukrohnia hamata) were plankton composition were grouped, although March and May samples were separated since season had a significant impact on the macrozooplankton patterns. Because of the low total abundance and biomass in MIX, these communities were treated separately, and divided into warm $\left(>1^{\circ} \mathrm{C}\right)$ and cold $\left(<0^{\circ} \mathrm{C}\right) \mathrm{MIX}$.

In terms of species richness, significantly fewer species were caught per replicate in MIX (warm and cold) and AtW in March than in ArW (bank and slope) and

Table 4. Summary of CA and CCA of macrozooplankton data from the Barents Sea marginal ice zone in May 1999 (spring) and March 2000 (late winter). (A) Seasonal variability included; (B) seasonal variability removed. Season accounted for $21 \%$ of the total macrozooplankton variability $(0.162 / 0.766 \times 100=21.1 \%)$

\begin{tabular}{|c|c|c|c|c|c|}
\hline \multirow{2}{*}{ Analysis } & \multicolumn{4}{|c|}{ - Axis } & \multirow{2}{*}{ Total inertia } \\
\hline & 1 & 2 & 3 & 4 & \\
\hline \multicolumn{6}{|l|}{ (A) Season incl. } \\
\hline Eigenvalue (CA) & 0.212 & 0.185 & 0.099 & 0.067 & 0.766 \\
\hline Eigenvalue (CCA) & 0.210 & 0.176 & 0.082 & 0.065 & 0.766 \\
\hline Species-environment correlation (CA) & 0.993 & 0.971 & 0.869 & 0.975 & \\
\hline Cumulative \% variance of species data (CA) & 27.7 & 51.9 & 64.9 & 73.7 & \\
\hline Cumulative $\%$ of species-environment relation (CA) & 34.5 & 63.2 & 75.5 & 86 & \\
\hline \multicolumn{6}{|l|}{ (B) Season excl. } \\
\hline Eigenvalue (CA) & 0.198 & 0.117 & 0.073 & 0.054 & 0.604 \\
\hline Eigenvalue (CCA) & 0.193 & 0.099 & 0.070 & 0.051 & 0.604 \\
\hline Species-environment correlation (CA) & 0.985 & 0.900 & 0.969 & 0.958 & \\
\hline Cumulative \% variance of species data (CA) & 32.8 & 52.1 & 64.2 & 73.2 & \\
\hline Cumulative $\%$ of species-environment relation (CA) & 43.4 & 64.3 & 79.7 & 90.8 & \\
\hline Seasonal variability & & & & & 0.162 \\
\hline
\end{tabular}


a)

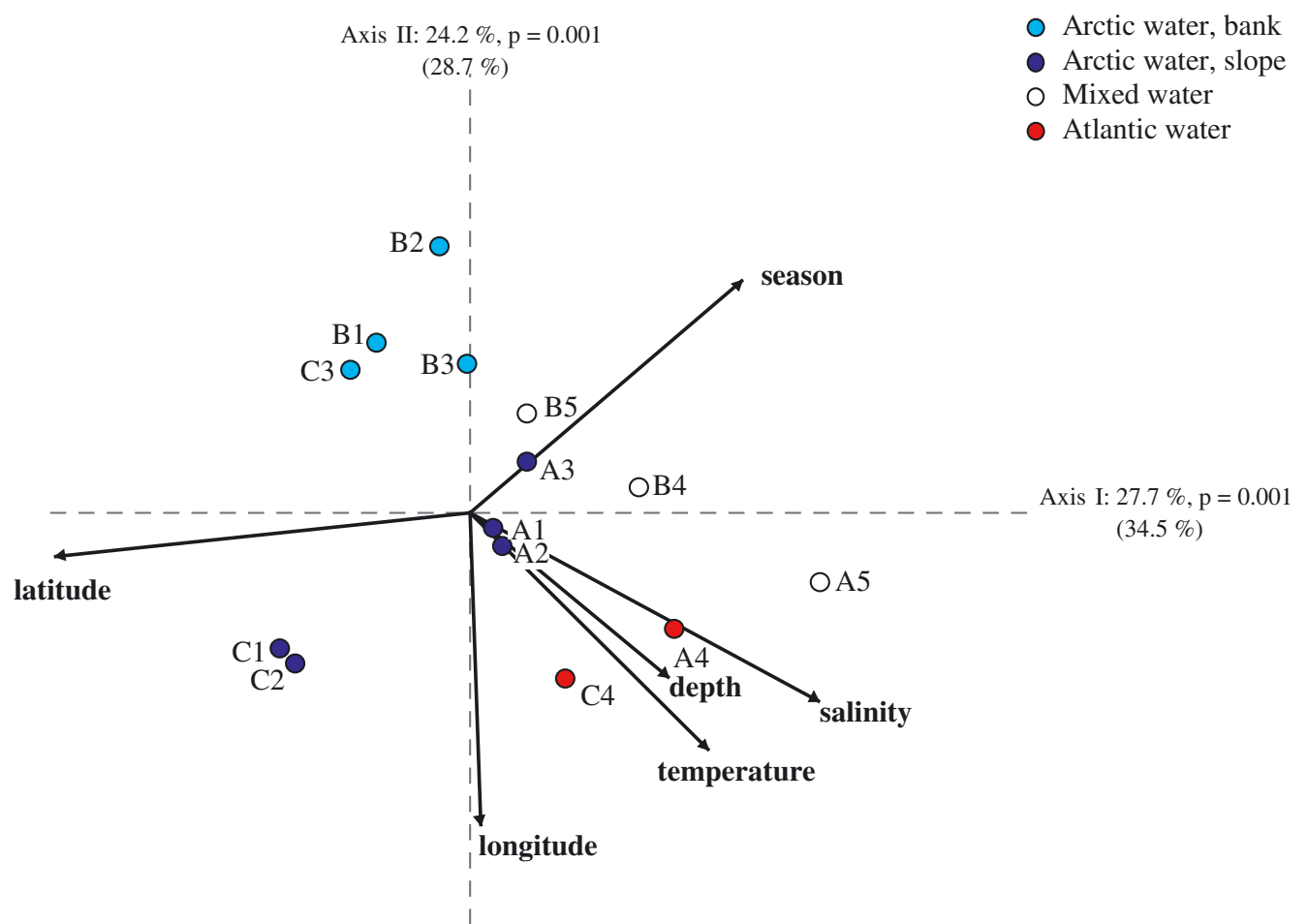

b)

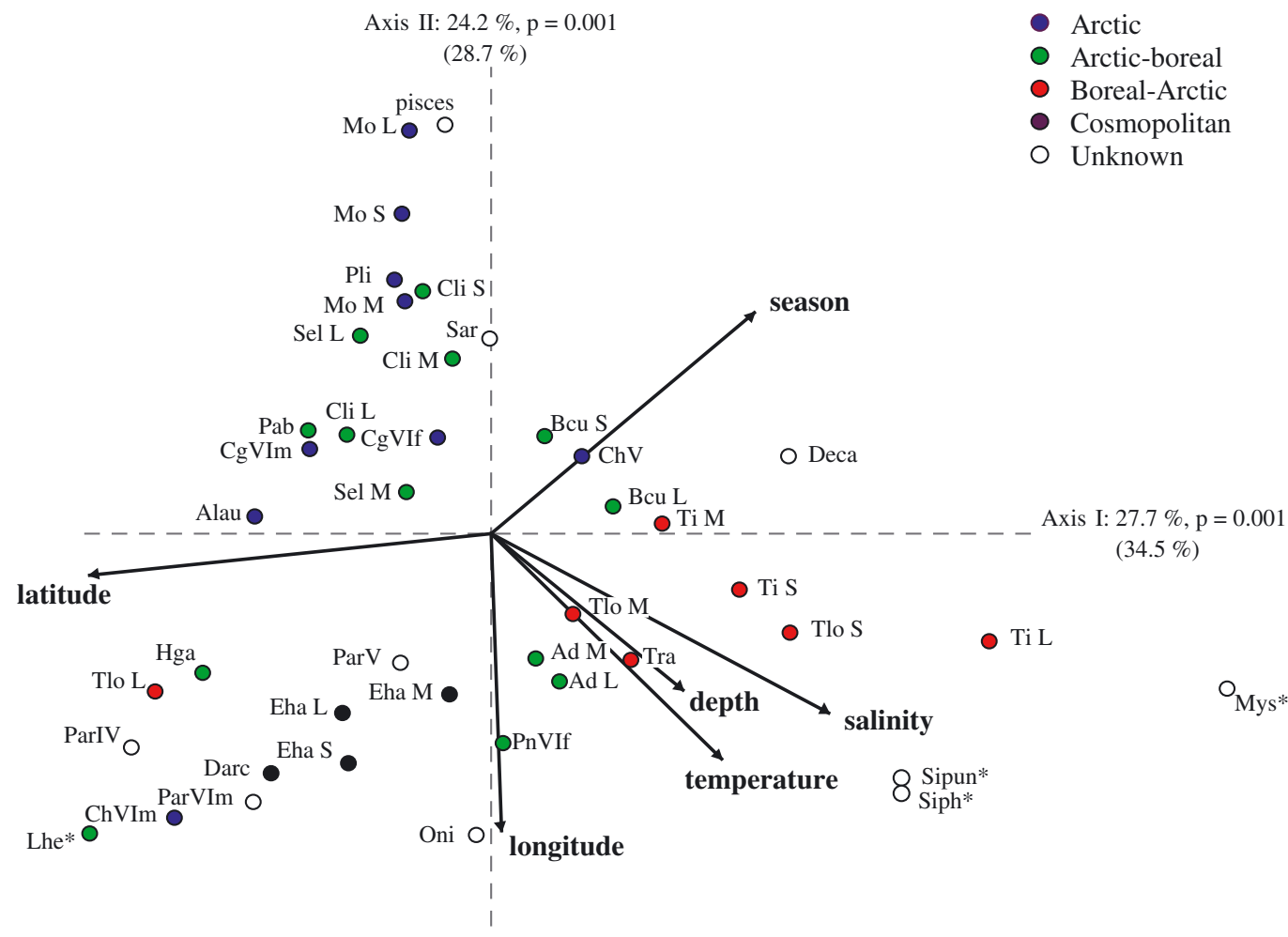

Fig. 5. Correspondence analysis (CA) (Axes I and II) of macrozooplankton abundance data describing their relationship to selected environmental variables (arrows) in the Barents Sea marginal ice zone during May 1999 (spring) and March 2000 (late winter). Plots explain $51.9 \%$ of total species variation and $63.2 \%$ of species-environment relationship (values in parentheses). Ordination of (a) stations and (b) taxa are displayed separately for clarity. Stations were classified according to dominating water mass, taxa were classified according to zoogeographical distribution. Abbreviations and literature sources as in Table 2. Taxa with distribution patterns $<15 \%$ explained by 2 -dimensional CA are not shown 
a) Axis II: $19.3 \%, \mathrm{p}=0.004$

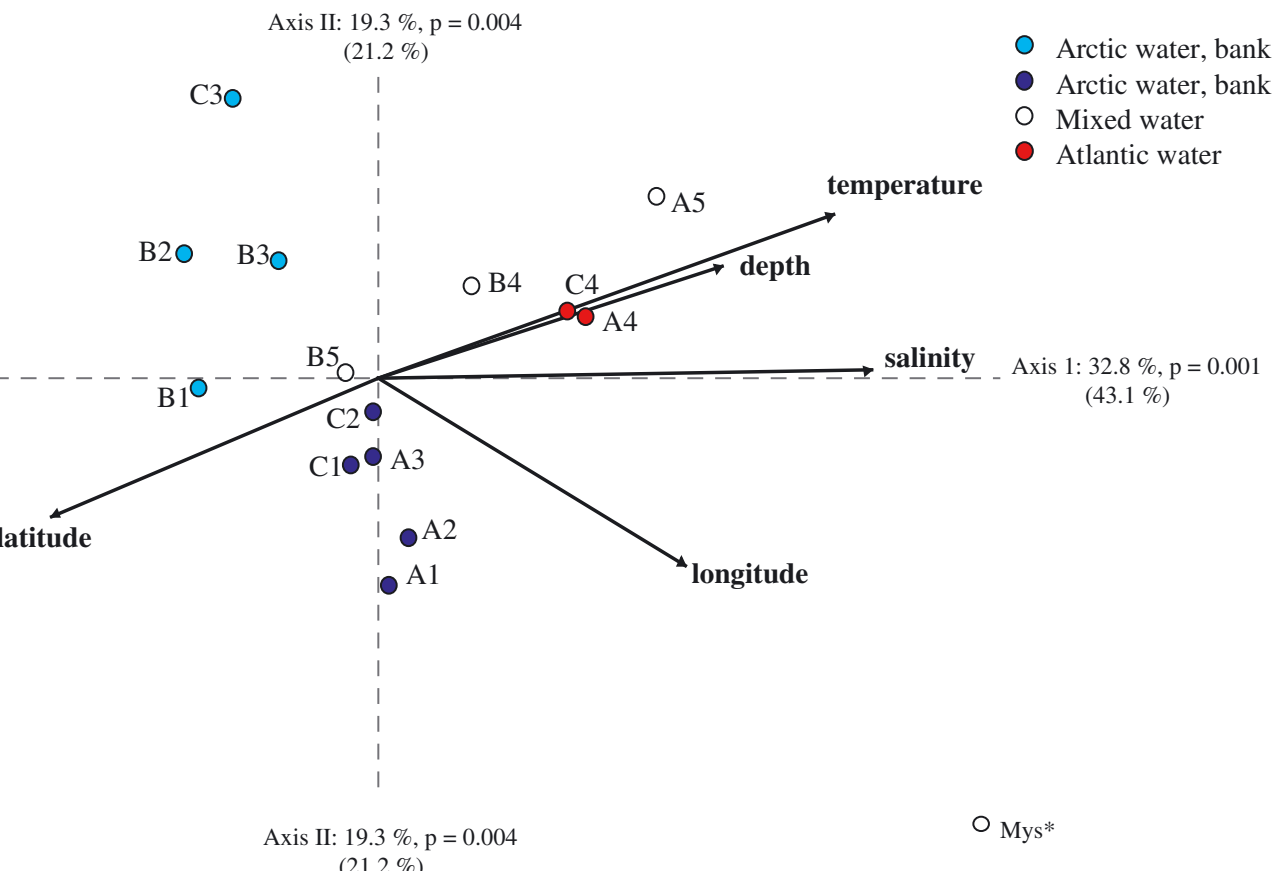

b)

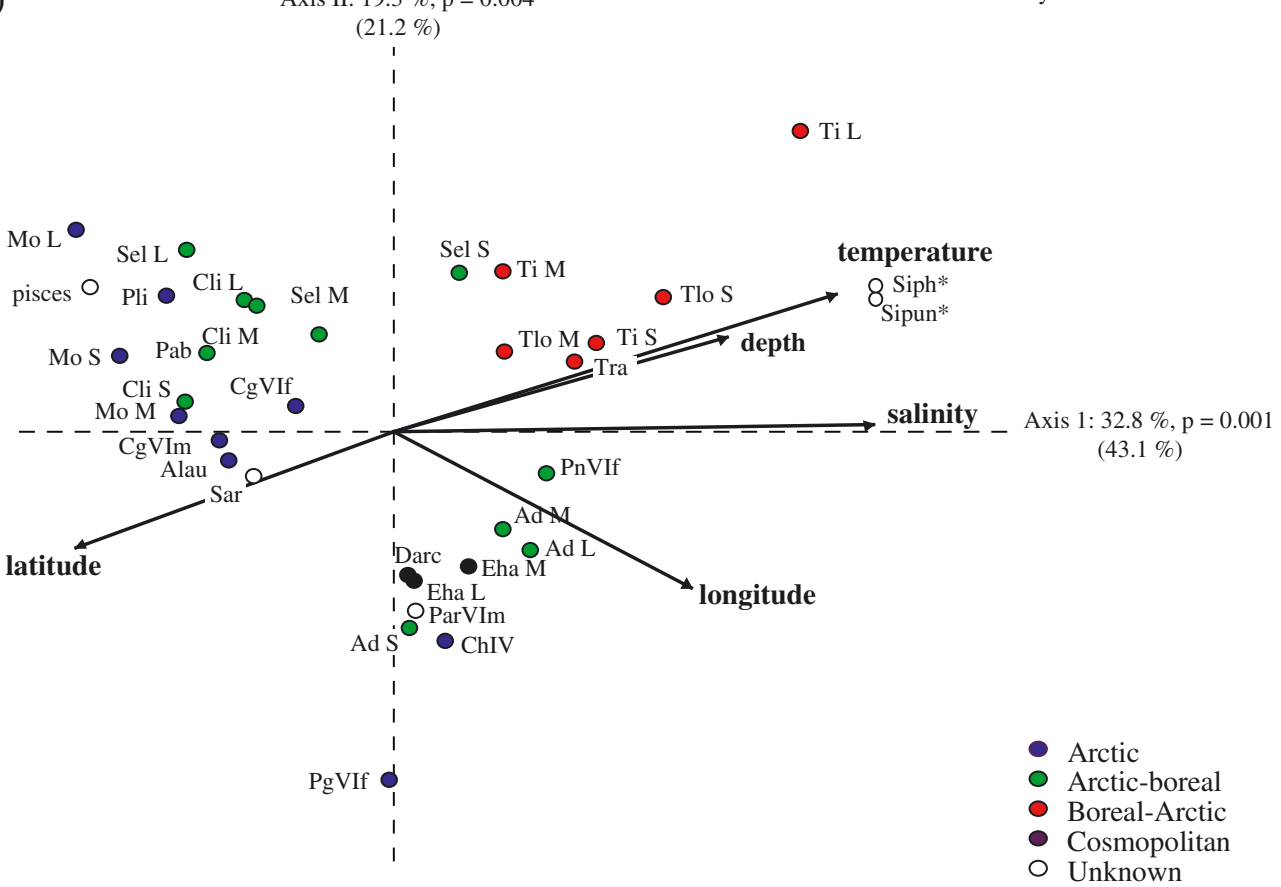

Fig. 6. Data from Fig. 5, with covariable month of sampling ('season') removed. Plots explain $52.1 \%$ of total species variation and $64.3 \%$ of species-environment relationship (in parentheses). Abbreviations as in Table 2

AtW in May (Fig. 7). A similar pattern was found for the total number of species: 12 to 14 species were found in MIX (warm and cold) and AtW in March, whereas 15 to 20 were found in ArW (bank and slope) and AtW in May.

Numerically, Calanus glacialis was most important north of the Polar Front (ArW), followed by C. hyperboreus (Fig. 8a). In AtW, C. hyperboreus was also important numerically, together with Thysanoessa inermis, but only in May. In March, the abundance of C. hyperboreus was low in AtW $\left(<2.5\right.$ ind. $\mathrm{m}^{-2}$ in all 3 replicates), whereas $T$. inermis was still relatively numerous, although its abundance varied greatly among the 3 replicates $\left(0.3,1.5\right.$ and 19 ind. $\left.\mathrm{m}^{-2}\right)$. The most abundant carnivorous species in the NW Barents Sea was Aglantha digitale (up to 31.2 ind. $\mathrm{m}^{-2}$ ), but 


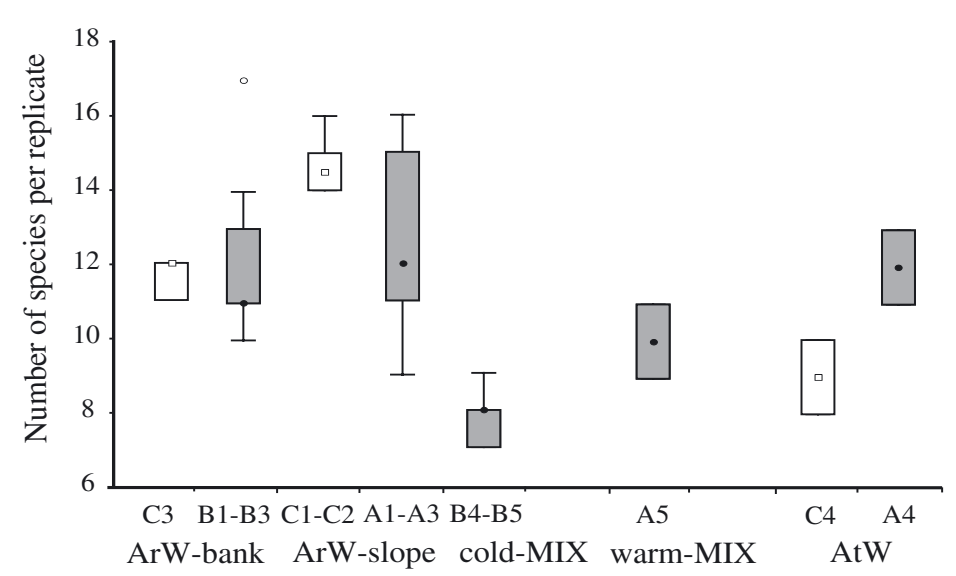

Fig. 7. Box plots of number of species sampled per replicate in macrozooplankton communities in the Barents Sea marginal ice zone during May 1999 (spring; grey boxes) and March 2000 (late winter; white boxes). Whiskers represent the non-outlier range and empty circles ( $\left.{ }^{\circ}\right)$ outliers. Water abbreviations as in Fig. 2
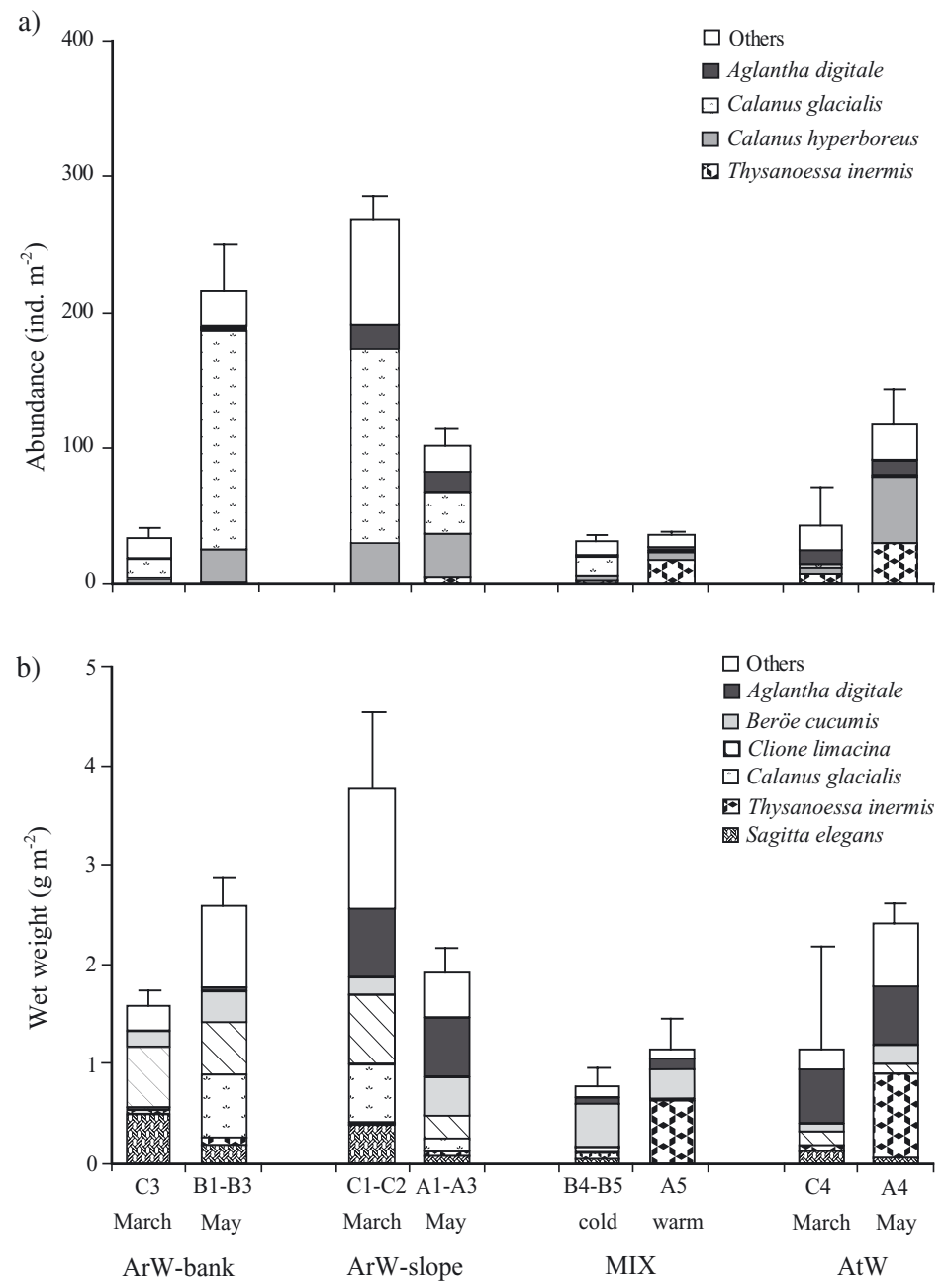

Fig. 8. (a) Abundance and (b) biomass of most important macrozooplankton species in sampling area in the Barents Sea marginal ice zone during May 1999 (spring) and March 2000 (late-winter). Error bars = SE of total. Water abbreviations as in Fig. 2 its abundance in MIX (cold and warm) and ArW bank waters was low $\left(<2.0\right.$ ind. $\left.\mathrm{m}^{-2}\right)$ (Table 2).

In terms of wet biomass, Beröe cucumis was important in all water masses sampled (Fig. 8b). Clione limacina contributed much to the total biomass north of the Polar Front, whereas Aglantha digitale and Thysanoessa inermis made up most of the biomass in AtW, except for $T$. inermis in March since only small juvenile individuals $(<16 \mathrm{~mm})$ were sampled at that time of the year in AtW. In May, a relatively high proportion of large and mature $T$. inermis (>22 mm) was sampled in warm-MIX compared to AtW, and the biomass of $T$. inermis was slightly higher in warm-MIX than in AtW. In May, the main biomass of Calanus glacialis was in the ArW bank waters, whereas in March the main biomass of this species was in ArW slope waters. The biomass of the carnivorous Sagitta elegans was 2 to 3 times larger in March than in May in ArW (bank and slope).

\section{Trophic composition and seasonal trends}

Herbivorous macrozooplankton dominated numerically in the northern Barents Sea, whereas the carnivores dominated in terms of wet biomass (Fig. 9). The abundance and biomass of herbivores increased from March to May in the ArW bank and AtW ( $\mathrm{p}<0.049)$, whereas no differences were found for the carnivores $(p>0.052)$. In ArW slope waters, the opposite was found. Here, the herbivores decreased in abundance and biomass from March to May $(p<0.003)$ as did the carnivores $(p=0.018)$, except in terms of biomass $(p=0.157)$.

In May, lower abundance of herbivores and carnivores were found in MIX (warm and cold) than in AtW and ArW (bank and slope) ( $p<0.049$ ) (Fig. 9). In biomass, the same was true, except for no differences in herbivorous biomass in the warm-MIX and ArW slope waters $(p=0.052)$. In March, herbivores and carnivores were found in similar low abundance and small biomass in the ArW bank and AtW ( $p>0.513$ ), whereas markedly higher abundance and larger biomass of herbivores $(p<0.01)$ and carnivores $(p<0.049)$ were found in the ArW slope (except for the carnivores in terms of biomass: $p>0.51$ ). 

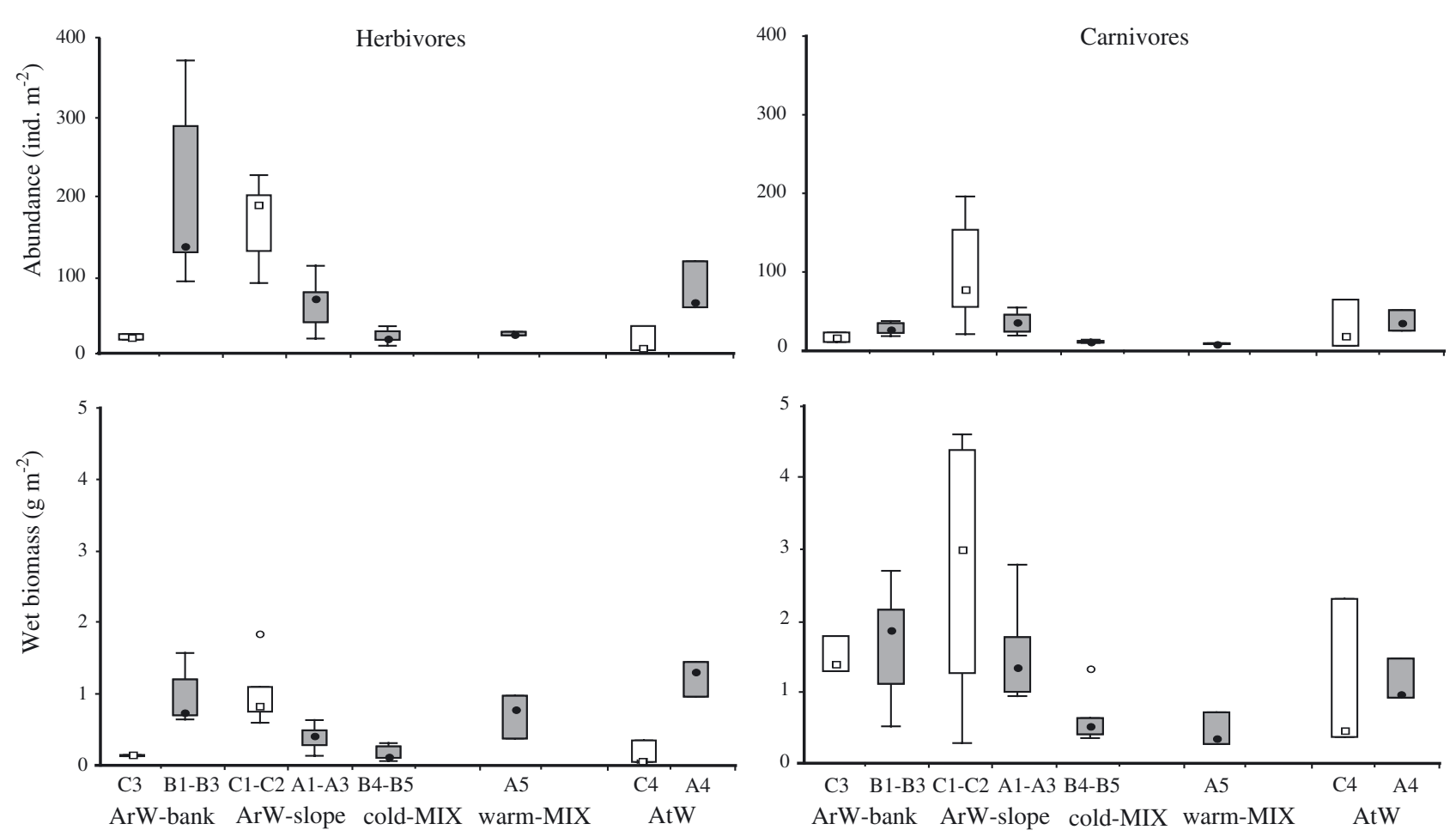

Fig. 9. Box plots of abundance and biomass of herbivores and carnivores in macrozooplankton communities sampled in the Barents Sea marginal ice zone during May 1999 (spring; grey boxes) and March 2000 (late-winter; white boxes). Whiskers represent the non-outlier range and empty circles $\left({ }^{\circ}\right)$ outliers. Water abbreviations as in Fig. 2

\section{DISCUSSION}

\section{Macrozooplankton variability}

A central question associated with plankton ecology is: How representative is the sampling in space and time? Replicate samples are the best way to address this question. In the present study, the variability in macrozooplankton abundance and biomass within stations was relatively low and did not exceed that between stations (Fig. 4). The spatial resolution was therefore sufficient to detect differences in horizontal macrozooplankton composition that were larger than those expected by chance. However, an exception was the highly variable macrozooplankton abundance and biomass in the 3 replicates in AtW in March. The reason for this variability is not known, since depth and sampled volume of water were similar and the same sampling procedure was followed in the 3 hauls. However, in March the AtW station (Stn C4) was located close to the Polar Front (Fig. 2) and, thus, hydrographical conditions could have changed during sampling, as the ship was adrift. But this variability could also be a result of patchiness, since there was no consistent decline or increase in total abundance during sampling of these 3 replicates (Hamner 1988). In particular, the number of krill (Thysanoessa spp.), which is known to form aggregations (Zelikman et al. 1978), varied largely among the 3 replicates.

Of special concern in regard to the estimates of macrozooplankton abundance and biomass was the error induced by net-selectivity. The macrozooplankton includes highly mobile organisms such as krill and amphipods, and visual avoidance of the net may have led to an underestimate of macrozooplankton abundance and biomass (Dalpadado \& Skjoldal 1991, Samoeto et al. 2000). Trawling is preferable for sampling the larger zooplankton fraction (Samoeto et al. 2000), but in dense pack-ice trawling is risky and often impossible. However, the large mouth opening $\left(2.01 \mathrm{~m}^{2}\right)$, the high filtration efficiency $(\sim 100 \%)$ and the relatively large volume of water filtered per station (500 to $2000 \mathrm{~m}^{3}$ ) probably minimised the sampling error arising from net avoidance (UNESCO 1968, Samoeto et al. 2000). However, the extrusion of smaller animals, may have been extensive (Samoeto et al. 2000). The abundance estimates of Calanus glacialis females may be $50 \%$ too low (see 'Materials and methods'); nevertheless, female C. glacialis were included in the analyses because their abundances were within the range of previously reported abundances in the MIZ in the Barents Sea (204 to 1557 ind. 
$\mathrm{m}^{-2}$; Falk-Petersen et al. 1999). However, the total abundance and biomass data for adult C. glacials and CVI of C. hyperboreus and Pareuchaeta spp. and for the other species in this study should be interpreted with caution because the total population was most probably not sampled (Fig. 3). The estimates of total abundance and biomass do not, however, affect the analyses of community- and species-environment relationships, since these were based on relative data.

\section{Environmental influences}

Only $\sim 20 \%$ of the total macrozooplankton variability was unexplained by the environmental variables latitude, longitude, salinity, temperature, bottom depth and season, indicating that these environmental variables are of great importance, or are possibly highly correlated with other important variables in determining the horizontal macrozooplankton distribution in the Barents Sea MIZ. Of greatest importance was water-mass distribution, followed by geographical position, season (late-winter vs spring) and bottom depth (Table 3). In spite of spatial and temporal limitations in the sampling design, our results are in good agreement with previous zooplankton studies in the Barents Sea, which indicate that water-mass distribution is of major importance in structuring the plankton communities in this region (Hassel 1986, Loeng 1989, Arashkevich et al. 2002). Gradients in zooplankton community and population structure from close pack-ice to open water have been recorded in the MIZ in the Barents Sea (Falk-Petersen et al. 1999), but whether this is a direct affect of the ice cover is not known. Quantitative studies of how several environmental factors affect, simultaneously, the zooplankton communities in the MIZ in the Arctic are lacking. In our study, ice-cover (close packice, open drift-ice and open water) and the stage of the associated spring bloom (Skjoldal \& Rey 1989, Hegseth 1998, Falk-Petersen et al. 2000a, Engelsen et al. 2002) had no influence on horizontal macrozooplankton structure. However, the sampling time period was limited and offspring were poorly sampled due to the selective sampling method. In addition, carnivorous taxa dominated the macrozooplankton communities, and these are generally less subject to seasonal food shortage than herbivorous zooplankton (Falkenhaug 1997, Hagen 1999). The Arctic herbivorous copepods Calanus glacialis and C. hyperboreus have also developed a variety of energetic adaptations and reproductive strategies that make them robust to seasonal variation in food availability. For instance, C. hyperboreus stores lipids, enabling this species to spawn during the dark season, and C. glacialis may utilise ice algae, which begin to grow as early as March in the Barents
Sea (Runge \& Ingram 1988, Conover \& Huntley 1991, Hegseth 1992). However, the stage of algal bloom (surface vs deep chlorophyll a maximum) may be important in determining the vertical distribution of macrozooplankton (e.g. Eilertsen et al. 1989, Unstad \& Tande 1991, Falkenhaug 1997).

In our study, salinity was the most important environmental variable determining the horizontal macrozooplankton distribution in the Barents Sea MIZ. However, salinity is more properly an indication of important advective processes. Experimental evidence suggests that a small salinity difference of 2.5 PSU, which exceeds the salinity difference between ArW and AtW, has no measurable effect on the physiology of zooplankters (McLaren et al. 1968, Kinne 1971). However, small changes in temperature may limit zooplankton distribution through altered growth, reproduction and/or mortality rates (McLaren 1969, Kinne 1970). The Barents Sea is a highly advective ecosystem, and many species, particularly those with centres of distribution in more temperate areas, may not successfully reproduce there. These expatriate populations will eventually become depleted, unless they are continually supplied by advected individuals (Pedersen 1995). In the northern Barents Sea, salinity is a better measure of AtW influence than temperature, because the temperature of a water mass is not only altered by water mixing, but also by atmospheric cooling during transport (Loeng 1991). Since our study used mean salinity and temperature of the dominant water mass at each station, the advective processes were not addressed properly. Latitude, longitude and bottom depth most probably identified some of the advective processes that our salinity and temperature variables did not describe. The latitudinal gradient was generally in good agreement with the salinity and temperature gradients, but exceptions were found because of the deep inflow of AtW between Nordaustlandet and Franz Josef Land and the strong impact of the bottom topography (Loeng 1991, Løyning 2000). Generally, ArW occurs in the bank areas in the northern Barents Sea, but over the shelf breaks (slopes) strong tidal currents force the heavier AtW up and onto the banks (Loeng 1991, Kowalik \& Proshutinsky 1995). This transport is particularly apparent over shallow banks (Kowalik \& Proshutinsky 1995), which explains the dominance of MIX over the shallow Spitsbergen Bank (Stns B4 and B5), relatively far from the shelf break (Fig. 2). A circular eddy that is topographically trapped may exist around the Great Bank (Løyning 2000), similar to the anticyclonic vortex around the Central Bank in the Barents Sea (Loeng 1989, Quadfasel et al. 1992). If this is so, the concentrating effect of this eddy, in addition to the inflow of Atlantic-derived waters from the north, would explain the relatively high faunal 
similarity over the northern and southern slopes of the Great Bank (Fig. 6). However, a relatively large seasonal difference in macrozooplankton composition was found within the ArW slope community (Fig. 5). The reasons for this difference may be cooling and/or, partly, the transformation of AtW on its way around Spitsbergen, and also the latitudinal distance between the northern and southern Great Banks (Fig. 1). At high latitudes, the solar angle, and thus the onset of spring, changes markedly over short latitudinal distances, especially in ice-covered waters (Sakshaug \& Slagstad 1991, Falk-Petersen et al. 2000a, Engelsen et al. 2002). In the Barents Sea MIZ, the spring bloom begins in mid-April to early May at the southernmost fringes of the first-year ice, and as late as August/ September close to the multiyear ice (Zenckevitch 1963, Sakshaug \& Slagstad 1991, Hegseth 1997). Latitudinal difference, as well as seasonal variability, was much less pronounced in the ArW bank and AtW macrozooplankton communities. It should be mentioned, however, that because season and latitude were moderately correlated, some of the latitudinal macrozooplankton variability might have been removed when adjusting for seasonal variability (ter Braak \& Smilauer 1998).

\section{Species-environment relationships}

In ArW bank, the most 'Arctic' environment, maximum abundances of the ctenophore Mertensia ovum (all sizes), the copepod Calanus glacialis (adults) and the amphipod Parathemisto libellula were found as expected (Figs. 5 \& 6) (Unstad \& Tande 1991, Hassel 1995, Dalpadado et al. 2001). The species that were most restricted to ArW were M. ovum and P. libellula, which were not recorded in warm-MIX or AtW during May 1999 and March 2000. However, relatively high abundances of small $M$. ovum $(<15 \mathrm{~mm})$ have been found south of the Polar Front in May (Swanberg \& Båmstedt 1991). In our study, mainly M. ovum $>15 \mathrm{~mm}$ were caught (Fig. 3). The second ctenophore, the Arctic-boreal Beröe cucumis, was widely distributed in the northern Barents Sea, but increased in abundance during spring (Fig. 8a). A possible predator-prey interaction has been suggested between B. cucumis and $M$. ovum (Greve 1981, Falk-Petersen et al. 2002). Above the Spitsbergen Bank, maximum numbers of small $B$. cucumis $(<20 \mathrm{~mm})$ coincided with maximum numbers of $M$. ovum (Table 2), which may indicate favourable feeding conditions for $B$. cucumis in that region (Hagen 1999). The abundance of $P$. libellula was very low in our study. High abundances of $P$. libellula (430 ind. $\mathrm{m}^{-2}$ ) have been found in May to June close to the Polar Front (Dalpadado et al. 2001), but most of these specimens ( 40 to $93 \%$ ) were 2 to $8 \mathrm{~mm}$. In our study, individuals $<2.5 \mathrm{~mm}$ were found in female brood-pouches, but were not counted. A southern (boreal) and a northern (Arctic) population of the pteropod Clione limacina co-exist in the Barents Sea (Mileikovsky 1970). In our study, small, medium and large individuals of C. limacina were confined to ArW bank, indicating that mainly individuals of the Arctic population occur in the Barents Sea MIZ. The chaetognath Sagitta elegans may also be comprised of 2 populations in the NW Barents Sea. S. elegans originating from more temperate regions have smaller maximum lengths than Arctic populations (McLaren 1969), which may explain the different environmental preferences of small- and large-sized S. elegans in this study.

In May, the Arctic copepod Calanus hyperboreus was found in high numbers both in ArW (bank and slope) and AtW. Seasonal migration and advective transport may explain the high abundance of $C$. hyperboreus in AtW in May and its low abundance there in March. In the Norwegian Sea, C. hyperboreus migrates to deep water (500 to $1500 \mathrm{~m}$ ) in autumn, i.e. to depths below those of the Barents Sea (Loeng 1991). However, in spring (April), it migrates upwards to feed, and is thus subjected to the North Atlantic current and transportation to the Barents Sea (Loeng 1991, Pedersen 1995, Hirche 1997).

In the relatively cold and saline waters of the ArW slope, the cosmopolitan species Dimophyes arctica and Eukrohnia hamata peaked in abundance together with the Arctic-boreal species Aglantha digitale and the cold-water species Pareuchaeta spp. These species are all widely distributed deep-water species (Bakke 1977, Sands 1980, Kirkpatrick \& Pugh 1984). They may not thrive in shallow bank areas or are less subject to advective transport into bank areas in the Barents Sea (Loeng 1991). A. digitale is considered to be a good indicator species of AtW (Mumm 1991, Hassel 1995). The similarly high abundance of this species in ArW slope and AtW can be a result of advection. However, the population of $A$. digitale in the Barents Sea may be of Arctic origin (Zelikman 1972). The ordination (Fig. 6b) showed that small A. digitale $(\leq 9 \mathrm{~mm}$ ) were less restricted to warm and saline waters than larger individuals ( $>9 \mathrm{~mm}$ ), which might support the idea that A. digitale is reproductively cold-adaptive. It is also possible that $A$. digitale reproduces later in ArW than in AtW. The siphonophore $D$. arctica was found in very low abundance in the NW Barents Sea, except for its particularly high abundance over the slopes of the NW Great Bank in March, where the hydromedusa Aeginopsis laurentii was also found in high abundance. Population bursts of $A$. laurentii and D. arctica can occur during warm years in the Barents Sea (Zelikman 1972). The year 1999 was characterised as 
warm, and the temperature continued to increase in 2000 (ICES 2000). The warmer temperatures may have had a positive impact on the populations of $D$. arctica and A. laurentii, since the previous years (1996 to 1998) were relatively cold (Ingvaldsen et al. 2002). The higher abundance of Pareuchaeta spp. and E. hamata, and also of Sagitta elegans in March compared to May might be explained by their life history. In Malangen, northern Norway, these 3 carnivores reach maximum abundances in October to February, and minimum abundances during summer (Falkenhaug 1997).

The boreal-Arctic Thysanoessa spp. showed, as expected, peak abundances in AtW (Dalpadado \& Skjoldal 1991). The distribution patterns of small, medium and large Thysanoessa spp. differed, particularly for $T$. longicaudata, but the differences were less extensive after seasonal variability had been removed. The largest individuals of $T$. longicaudata ( $>15 \mathrm{~mm}$ ) were found in ArW, corresponding to the findings of Dalpadado \& Skjoldal (1991). T. longicaudata probably grows to a larger size and reaches an older age in cold ArW than in warmer AtW (Dalpadado \& Skjoldal 1996). Large $T$. inermis (>22 mm) were only found in May in the Hopen Trench. However, large $T$. inermis could also have been present there in March, as they may live close to the bottom during the winter months (Kulka \& Corey 1978, Lindley 1980, Dalpadado \& Skjoldal 1995) and would therefore not have been sampled during our study. Only 2 individuals of the euphausiid Meganyctiphanes norvegica were found during May 1999 and March 2000, confirming the more boreal distribution of this species compared to Thysanoessa spp. Rarely occurring taxa were mainly restricted to AtW and were probably expatriate species transported into the Barents Sea.

\section{Macrozooplankton species diversity, abundance and biomass}

The abundance of macrozooplankton in the MIZ in the Barents Sea was low, approximately 40 times lower than the recorded abundance of meso-zooplankton $(>180 \mu \mathrm{m})$ in the same area in May 1999 (S. Kwasniewski et al. unpubl.). Oceanic fronts are generally connected with enhanced biological production (Mann \& Lazier 1996), and this has also been suggested for the Polar Front in the Barents Sea (Loeng 1989, Mehlum et al. 1998). However, in May 1999, the abundance, biomass and number of macrozooplankton species were particularly low in the frontal areas (MIX) compared to those in AtW and ArW (except for in AtW in March). The low values suggest that the Polar Front is not only a physical border but also a biological border for many macrozooplankton species. However, most studies from the Barents Sea report a high biomass of macrozooplankton in summer and late autumn, but a low biomass in spring (Zelikman \& Kamshilov 1960, Hassel 1986, Loeng 1989). The macrozooplankton abundance and biomass in MIX might therefore increase during summer. Usually, AtW is connected with higher abundance and biomass than ArW (Mumm et al. 1998). However, in this study the stations dominated by AtW were located at the extreme northern end of the AtW influence. AtW normally maintains a temperature of 3 to $6^{\circ} \mathrm{C}$ close to Bear Island, but cools on its way north (Loeng 1991). During May 1999 and March 2000, the AtW was 1 to $1.5^{\circ} \mathrm{C}$ in the inner Hopen Trench. This gradual cooling along with the increasing distance from the Norwegian Sea result in rare occurrences of boreal species in the northern Barents Sea (Loeng 1989).

Some differences in macrozooplankton abundance and biomass were not readily explained by water-mass distribution alone, such as the particularly low abundance and biomass of herbivores in the ArW bank in March compared to May (Fig. 9). Seasonal adaptations are closely related to the trophic level of zooplankters, and the life-cycle strategies of the herbivorous Calanus glacialis and C. hyperboreus include seasonal vertical migrations and diapause (Conover \& Huntley 1991, Hagen 1999). Calanus spp. that descend for overwintering, 'stream' down to deeper parts of the shelf and into trenches, where they concentrate close to the bottom (Kashkin 1962). Seasonal migration patterns most probably explain the variability in species abundance and biomass within the ArW slope and ArW bank communities. Carnivorous macrozooplankton also increased in abundance in ArW slope in winter, although their biomass did not significantly increase. However, carnivores usually feed year-round, and may therefore follow the seasonal migrations of their copepod prey (Øresland 1990, Falkenhaug 1997, Hagen \& Auel 2001). The much lower abundance of herbivores in AtW in March than in AtW in May can be explained by seasonal migrations and advective transport (as for C. hyperboreus described above). However, the particularly small biomass of herbivores, was also a result of the absence of large Thysanoessa inermis ( $\geq 16 \mathrm{~mm})$ in AtW in March.

Large-scale water-mixing in the NW Barents Sea leads to communities characterised by differences in species abundance and biomass rather than differences in taxonomic composition. Both the physical and biological environment alter a species survival, growth and generation time. It is therefore important in future studies to compare developmental stages and/or size classes rather than species as one unit when studying community-environment relationships. Increased 
knowledge of species' ecology, i.e. their trophic levels, population dynamics and limiting physical and biological factors (e.g. temperature, competition and predation pressure) is essential in order to better understand the spatial and temporal variations in zooplankton abundance and biomass.

Acknowledgements. We thank J. Wiktor and E. N. Hegseth for the chlorophyll a data, and the captain and crew of RV 'Lance' for ship logistics and assistance in the field. We further thank the Norwegian Polar Institute, Tromsø, the Institute of Oceanology, Sopot and the partners of the Barents Sea Production Licenses 182, 225 and 228 (Norsk Hydro, Statoil, Agip, Chevron, Fortum, Petero and Enterprise-contract no. 900000465) for financial and technical support.

\section{LITERATURE CITED}

Arashkevich E, Wassmann P, Pasternak A, Wexels Riser C (2002) Seasonal and spatial changes in biomass, structure and development progress of zooplankton community in the Barents Sea. J Mar Syst 38:125-145

Bakke JLW (1977) Ecological studies on the deep-water pelagic community of Korsfjorden, western Norway. Population dynamics of Euchaeta norvegica (Crustacea, Copepoda) from 1971 to 1974. Sarsia 63:49-55

Bray JR, Curtis JT (1957) An ordination of the upland forest communities of southern Wisconsin. Ecol Monogr 27: 325-349

Clarke A (1983) Life in cold water: the physiological ecology of polar marine ectotherms. Oceanogr Mar Biol Annu Rev 21:341-453

Clarke A (1988) Seasonality in the Antarctic marine environment. Comp Biochem Physiol B 90:463-471

Clarke KR, Gorley RN (2001) PRIMER v5: user manual/ tutorial. PRIMER-E, Plymouth

Clarke KR, Warwick RM (1994) Change in marine communities: an approach to statistical analysis and interpretation. Plymouth Marine Laboratory, Plymouth

Colin OH, Satterlie A (1992) Fast-strike feeding behavior in a pteropod mollusk, Clione limacina Phipps. Biol Bull (Woods Hole) 182:1-7

Conover RJ, Huntley M (1991) Copepods in ice-covered seas-distribution, adaptions to seasonally limited food, metabolism, growth patterns and life cycle strategies in polar seas. J Mar Syst 2:1-41

Dalpadado P, Skjoldal HR (1991) Distribution and life history of krill from the Barents Sea. Polar Res 10:443-460

Dalpadado P, Skjoldal HR (1995) Distribution and life cycle of krill north of $73^{\circ} \mathrm{N}$ in the Barents Sea, 1984-1992. Fisken Havet 16:1-50

Dalpadado P, Skjoldal HR (1996) Abundance, maturity and growth of the krill species Thysanoessa inermis and $T$. longicaudata in the Barents Sea. Mar Ecol Prog Ser 144: 175-183

Dalpadado P, Borkner N, Bogstad B, Mehl S (2001) Distribution of Themisto (Amphipoda) spp. in the Barents Sea and predator-prey interactions. ICES J Mar Sci 58:876-895

Dawson JK (1978) Vertical distribution of Calanus hyperboreus in the central Arctic Ocean. Limnol Oceanogr 23: 350-357

Dittrich BU (1992) Functional morphology of the mouthparts and feeding strategies of the parasitic amphipod Hyperia galba (Montagu, 1813). Sarsia 77:11-18
Dunbar MJ (1954) The amphipod Crustacea of Ungava Bay, Canadian eastern Arctic. J Fish Res Board Can 11:709-798

Dunbar MJ (1964) Serial atlas of the marine environment, Folio 6: euphausiids and pelagic amphipods. American Geographical Society, New York

Eilertsen HC, Tande KS, Taasen JP (1989) Vertical distributions of primary production and grazing by Calanus glacialis Jaschnov and Calanus hyperboreus Krøyer in Arctic waters (Barents Sea). Polar Biol 4:253-260

Einarsson H (1945) Euphausiacea 1: northern Atlantic species. Dana Rep no. 27

Ekman S (1953) Zoogeography of the sea. Sidgwick \& Jackson, London

Engelsen O, Hegseth EN, Hop H, Hansen E, Falk-Petersen S (2002) Spatial variability of chlorophyll $a$ in the marginal ice zone of the Barents Sea, with relations to sea ice and oceanographic conditions. J Mar Syst 35:79-97

Falkenhaug T (1991) Prey composition and feeding rate of Sagitta elegans var. arctica (Chaetognatha) in the Barents Sea in early summer. Polar Res 10:487-506

Falkenhaug T (1997) Studies of spatio-temporal variations in a zooplankton community: interactions between vertical behaviour and physical processes. Dr. Sci thesis, Norwegian College of Fishery Science, University of Tromsø

Falk-Petersen S, Pedersen G, Kwasniewski S, Hegseth EN, Hop H (1999) Spatial distribution and life-cycle timing of zooplankton in the marginal ice zone of the Barents Sea during the summer melt season in 1995. J Plankton Res 21: 1249-1264

Falk-Petersen S, Hop H, Budgell WP, Hegseth EN and 5 others (2000a) Physical and ecological processes in the marginal ice zone of the northern Barents Sea during the summer melt period. J Mar Syst 27:131-159

Falk-Petersen S, Hagen W, Kattner G, Clarke A, Sargent J (2000b) Lipids, trophic relationships, and biodiversity in Arctic and Antarctic krill. Can J Fish Aquat Sci 57:178-191

Falk-Petersen S, Dahl TM, Scott CL, Sargent JR, Gulliksen B, Kwasniewski S, Hop H, Millar RM (2002) Lipid biomarkers and trophic linkages between ctenophores and copepods in Svalbard waters. Mar Ecol Prog Ser 227:187-194

Gilmer RW, Harbison GR (1991) Diet of Limacina helicina (Gastropoda: Techostomata) in Arctic waters in midsummer. Mar Ecol Prog Ser 77:125-134

Greve W (1981) Invertebrate predator control in a coastal marine ecosystem: the significance of Beröe gracilis (Ctenophora). Kiel Meeresforsch Sonderh 5:211-217

Hagen W (1999) Reproductive strategies and energetic adaptations of polar zooplankton. Invertebr Reprod Dev 36: 25-34

Hagen W, Auel H (2001) Seasonal adaptions and the role of lipids in oceanic zooplankton. Zoology 104:313-326

Hamner WM (1988) Behavior of plankton and patch formation in pelagic ecosystems. Bull Mar Sci 43:752-757

Hamre J (1994) Biodiversity and exploitation of the main fish stocks in the Norwegian-Barents Sea ecosystem. Biodiv Conserv 3:473-492

Harding GCH (1974) The food of deep-sea copepods. J Mar Biol Assoc UK 54:141-155

Harris CL, Plueddemann AJ, Gawarkiewicz GG (1998) Water mass distribution and polar front structure in the western Barents Sea. J Geophys Res C Oceans 103:2905-2917

Hassel A (1986) Seasonal changes in zooplankton composition in the Barents Sea, with special attention to Calanus spp. (Copepoda). J Plankton Res 8:329-339

Hassel A (1995) Dyreplankton i Barentshavet: biomasse-variasjoner 1979-1994. Fisken Havet 1:1-38 (English summary) Hassel A, Skjoldal HR, Gjøsæter H, Loeng H, Omli L (1991) 
Impact of grazing from capelin (Mallotus villosus) on zooplankton: a case study in the northern Barents Sea in August 1985. Polar Res 10:371-388

Hegseth EN (1992) Sub-ice algal assemblages of the Barents Sea: Species composition, and growth rates. Polar Biol 12: 485-496

Hegseth EN (1997) Phytoplankton of the Barents Sea-the end of the growth season. Polar Biol 17:235-241

Hegseth EN (1998) Primary production of the northern Barents Sea. Polar Res 17:113-123

Hirche HJ (1997) Life cycle of the copepod Calanus hyperboreus in the Greenland Sea. Mar Biol 128:607-618

Hop H, Falk-Petersen S (2003) Temporal and spatial variability of the ice-ocean system of the ice edge in the marginal ice zone of the Barents Sea. MARINØK cruises, May 1999 and March-April 2000. Nor Polarinst Internrapp no. 11 (available from Norwegian Polar Institute)

ICES (International Council for the Exploration of the Sea) (2000) The annual ICES ocean climate status summary 1999/2000. ICES, Copenhagen, available at http://www. ices.dk/status/clim9900/

Ingvaldsen R, Loeng H, Asplin L (2002) Variability in the Atlantic inflow to the Barents Sea based on a one-year time series from moored current meters. Cont Shelf Res 22:505-519

Jongman RHG, ter Braak CJF, van Tongeren OFR (1995) Data analysis in community and landscape ecology. Cambridge University Press, Cambridge

Kaltin S, Anderson LG, Olsson K, Fransson A, Chierici M (2002) Uptake of atmospheric carbon dioxide in the Barents Sea. J Mar Syst 38:31-45

Kashkin NI (1962) On the adaptive value of seasonal migrations of Calanus finmarchicus (Gunnerus, 1770). Zool Zh 41:342-357 (in Russian with English summary)

Kinne O (1970) Temperature, animals: invertebrates. In: Kinne O (ed) Marine ecology, Vol 1. Environmental factors, Part 1. Wiley-Interscience, London, p 407-514

Kinne O (1971) Salinity, animals: invertebrates. In: Kinne O (ed) Marine ecology, Vol 1. Environmental factors, Part 2. Wiley-Interscience, London, p 821-995

Kirkpatrick PA, Pugh PR (1984) Siphonophores and velellids: keys and notes for the identification of the species. Synop Br Fauna New Ser 29:

Klekowski RZ, Weslawski JM (eds) (1991) Atlas of the marine fauna of southern Spitsbergen, Vol 2. Institute of Oceanology, Gdansk

Kowalik Z, Proshutinsky AY (1995) Topographic enhancement of tidal motion in the western Barents Sea. J Geophys C Oceans Res 100:2613-2637

Kulka DW, Corey S (1978) The life history of Thysanoessa inermis (Krøyer) in the Bay of Fundy. Can J Zool 56: 492-506

Kwasniewski S, Hop H, Falk-Petersen S, Pedersen G (2003) Distribution of Calanus species in Kongsfjorden, a glacial fjord in Svalbard. J Plankton Res 25:1-20

Lindley JA (1980) Population dynamics and production of euphausiids. II. Thysanoessa inermis and T. raschii in the North Sea and American coastal waters. Mar Biol 59: $225-233$

Loeng H (1989) Ecological features of the Barents Sea. In: Rey L, Alexander V (eds) Proc 6th Conf Com Arctique Int, 13-15 May 1985. EJ Brill, Leiden, p 327-365

Loeng H (1991) Features of the physical oceanographic conditions of the Barents Sea. Polar Res 10:5-18

Longhurst AR (1976) Vertical migration. In: Cushing DH, Walsh JJ (eds) The ecology of the seas. Blackwell, Oxford, p 116-139
Løyning TB (2000) Hydrography in the north-western Barents Sea, July-August 1996. Polar Res 20:1-12

Madin LP (1988) Feeding behaviour in tentaculata predators: in situ observations and conceptual model. Bull Mar Res 43:413-429

Mann KH, Lazier JRN (1996) Dynamics of marine ecosystems. Blackwell, Oxford

Matthews JBL, Hestad L (1977) Ecological studies on the deep-water pelagic community of Korsfjorden, western Norway: length/weight relationships for some macroplanctonic organisms. Sarsia 63:57-63

McLaren IA (1969) Population and production ecology of zooplankton in Ogac Lake, a landlocked fjord on Baffin Island. J Fish Res Board Can 26:1485-1559

McLaren IA, Walker DA, Corkett CJ (1968) Effects of salinity on mortality and development rate of eggs of the copepod Pseudocalanus minutes. Can J Zool 46:1267-1269

Mehlum F, Nordlund N, Isaksen K (1998) The importance of the 'Polar Front' as a foraging habitat for guillemots Uria spp. breeding at Bjørnøya, Barents Sea. J Mar Syst 14: $27-43$

Mileikovsky SA (1970) Breeding and larval distribution of the pteropod Clione limacina in the North Atlantic, subarctic and North Pacific Oceans. Mar Biol 6:317-334

Mumm N (1991) Zur sommerlichen Verteilung des Mesozooplanktons im Nansen-Becken, Nordpolarmeer. Ber Polarforsch 92 (English summary)

Mumm N, Auel H, Hanssen H, Hagen W, Richter C, Hirche HJ (1998) Breaking the ice: large-scale distribution of mesozooplankton after a decade of Arctic and transpolar cruises. Polar Biol 20:189-197

Nilssen KT, Haug T, Potelov V, Timoshenko YK (1995) Feeding-habits of harp seals (Phoca groenlandica) during early summer and autumn in the northern Barents Sea. Polar Biol 15:485-493

NOAA (National Oceanic and Atmospheric Administration) (2001) Observer's guide to sea ice. US Department of Commerce, Seattle, available at http://www.response. restoration.noaa.gov/oilaids/seaice/seaice.html

Øresland V (1990) Feeding and predation impact of the chaetognath Eukrohnia hamata in Gerlache Strait, Antarctic Peninsula. Mar Ecol Prog Ser 63:201-209

Østvedt OJ (1955) Zooplankton investigations from weathership ' $\mathrm{M}$ ' in the Norwegain Sea, 1948-49. Hvalråd Skr 40:1-93

Pedersen G (1995) Factors influencing the size and distribution of the copepod community in the Barents Sea with special emphasis on Calanus finmarchicus (Gunnerus). Dr. Sci thesis, Norwegian College of Fishery Science, University of Tromsø

Pedersen G, Tande KS, Nilssen EM (1995) Temporal and regional variation in the copepod community in the central Barents Sea during spring and early summer 1988 and 1989. J Plankton Res 17:263-282

Pierrot-Bults AC, Chidgey KC (1988) Chaetognatha: keys and notes for the identification of the species. Synopses of the British Fauna. New series no. 39

Quadfasel D, Rudels B, Selchow S (1992) The Central Bank vortex in the Barents Sea: watermass transformation and circulation. ICES Mar Sci Symp 195:40-51

Rasmussen R (1971) Om biologien til Aglantha digitale (O. F. Müller) med bemerkninger om forekomsten av de øvrige hydromeduser i Korsfjorden, Vest Norge. Cand real thesis, University of Bergen

Runge JA, Ingram RG (1988) Under-ice grazing by planktonic calanoid copepods in relation to a bloom of ice microalgae in southeastern Hudson Bay. Limnol Oceanogr 33:280-286 
Sakshaug E, Slagstad D (1991) Light and productivity of phytoplankton in polar marine ecosystems: a physiological view. Polar Res 10:69-85

Sakshaug E, Bjørge A, Gulliksen B, Loeng H, Mehlum F (1994) Structure, biomass distribution, and energetics of the pelagic ecosystem in the Barents Sea: a synopsis. Polar Biol 14:405-411

Sameoto D, Wiebe P, Runge J, Postel L, Dunn J, Miller C, Coombs S (2000) Collecting zooplankton. In: Harris RP, Wiebe PH, Lenz J, Skjoldal HR, Huntley M (eds) Zooplankton methodology manual. Academic Press, London, p 53-81

Sands NJ (1980) Ecological studies on the deep-water community of Korsfjorden, western Norway: population dynamics of the chaetognaths from 1971-1974. Sarsia 65:1-12

Skjoldal HR, Rey F (1989) Pelagic production and variability of the Barents Sea ecosystem. In: Sherman K, Alexander LM (eds) Biomass yields and geography of large marine ecosystems. AAAS selected symposium 111. Westview Press, Washington, p 241-286

Skjoldal HR, Hassel A, Rey F (1987) Spring phytoplankton development and zooplankton reproduction in the central Barents Sea in the period 1979-1984. In: Loeng H (ed) The effect of oceanographic conditions on distribution and population dynamics of commercial fish stocks in the Barents Sea. Proc 3rd Sov-Norw Symp, Murmansk, May 26-28, 1986, Institute of Marine Research, Bergen, p 59-89

Swanberg N, Båmstedt U (1991) Ctenophora in the Arctic: the abundance, distribution and predatory impact of the cydippid ctenophore Mertensia ovum (Fabricius) in the Barents Sea. Polar Res 10:507-524

Tande KS, Henderson RJ (1988) Lipid composition of copepodite stages and adult females of Calanus glacialis in Arctic waters of the Barents Sea. Polar Biol 8:333-339

Tande KS, Hassel A, Slagstad D (1985) Gonad maturation and possible life cycle strategies in Calanus finmarchicus and

Editorial responsibility: Otto Kinne (Editor),

Oldendorf/Luhe, Germany
Calanus glacialis in the northern Barents Sea. In: Gray JS, Christiansen ME (eds) Marine biology of polar regions and effects of stress on marine organisms. John Wiley \& Sons, Chichester

ter Braak CJF, Smilauer P (1998) CANOCO reference manual and user's guide to Canoco for Windows: software for canonical community ordination (version 4). Microcomputer Power, Ithaca, NY

ter Braak CJF, Verdonschot PFM (1995) Canonical correspondence analysis and related multivariate methods in aquatic ecology. Aquat Sci 57:255-289

UNESCO Press (1968) Zooplankton sampling. UNESCO, Paris

Unstad KH, Tande KS (1991) Depth distribution of Calanus finmarchicus and C. glacialis in relation to environmental conditions in the Barents Sea. Polar Res 10:409-420

Vinje T, Kvambekk AS (1991) Barents Sea drift ice characteristics. Polar Res 10:59-68

Wiborg KF (1954) Investigations on zooplankton in coastal and offshore waters off western and northwestern Norway, with special reference to the copepods. Fiskeridir Skr Ser Havunders no. 9

Zelikman EA (1972) Distribution and ecology of the pelagic hydromedusae, siphonophores and ctenohores of the Barents Sea, based on perennial plankton collections. Mar Biol 17:256-264

Zelikman EA, Kamishilov MM (1960) The long-term dynamics of plankton biomass in the southern part of the Barents Sea, and factors influencing it. Tr Murm Morsk Biol Inst 2:68-113 (in Russian)

Zelikman EA, Lukashevich IP, Drobysheva SS (1978) Aggregative distribution of Thysanoessa inermis (Kröyer) $T$. raschii (M. Sars) (Euphausiacea) in the Barents Sea. Oceanology 18:709-713

Zenckevitch L (1963) Biology of the seas of the USSR. George Allen \& Unwin, London

Submitted: October 4, 2002; Accepted: August 7, 2003

Proofs received from author(s): November 14, 2003 